\title{
In search of the focus of attention in working memory: 13 years of the retro-cue effect
}

\author{
Published online: 20 April 2016 \\ (C) The Psychonomic Society, Inc. 2016
}

Alessandra S. Souza ${ }^{1}$ - Klaus Oberauer ${ }^{1}$

\begin{abstract}
The concept of attention has a prominent place in cognitive psychology. Attention can be directed not only to perceptual information, but also to information in working memory (WM). Evidence for an internal focus of attention has come from the retro-cue effect: Performance in tests of visual WM is improved when attention is guided to the testrelevant contents of WM ahead of testing them. The retro-cue paradigm has served as a test bed to empirically investigate the functions and limits of the focus of attention in WM. In this article, we review the growing body of (behavioral) studies on the retro-cue effect. We evaluate the degrees of experimental support for six hypotheses about what causes the retro-cue effect: (1) Attention protects representations from decay, (2) attention prioritizes the selected WM contents for comparison with a probe display, (3) attended representations are strengthened in WM, (4) not-attended representations are removed from WM, (5) a retro-cue to the retrieval target provides a head start for its retrieval before decision making, and (6) attention protects the selected representation from perceptual interference. The extant evidence provides support for the last four of these hypotheses.
\end{abstract}

Keywords Attention $\cdot$ Retro-cue $\cdot$ Working memory

Alessandra S. Souza

a.souza@psychologie.uzh.ch

Klaus Oberauer

k.oberauer@psychologie.uzh.ch

1 Department of Psychology, Cognitive Psychology Unit, University of Zurich, Binzmühlestrasse 14/22, 8050 Zurich, Switzerland
An information-processing system faces two big challenges: the creation of processing priorities among relevant pieces of information, and the selection of relevant input amidst irrelevant ones. Attention is assumed to serve both of these functions for our cognitive system (Carrasco, 2011; Yantis, 2008). Attention can be directed both to information coming through the senses, and to information generated or maintained internally in the absence of corresponding perceptual input (Chun, Golomb, \& Turk-Browne, 2011). Notwithstanding the theoretical relevance of attention in both domains, attentional effects are far better understood in the perceptual domain (see the reviews by Carrasco, 2011; Egeth \& Yantis, 1997; Serences $\&$ Yantis, 2006) than in the realm of internal information.

In the present review, we aim to bring to focus the research on how attention affects internal information, and in particular, information in working memory (WM). WM is the system enabling online maintenance of representations for ongoing cognition. Accordingly, performance in WM tasks is associated with several measures of complex cognition, such as reading comprehension, reasoning, intelligence, and educational outcomes (e.g., Bull, Espy, \& Wiebe, 2008; Conway, Kane, \& Engle, 2003; Daneman \& Carpenter, 1980; Süß, Oberauer, Wittmann, Wilhelm, \& Schulze, 2002). Despite its central role for higher cognition, WM storage capacity is severely limited: Only a few representations at a time can be maintained accessible (Cowan, 2010). Moreover, representations in WM are not all in the same state of accessibility, and not all are equally relevant for the current task goals (Oberauer \& Hein, 2012; Olivers, Peters, Houtkamp, \& Roelfsema, 2011). Investigating how an attentional set can be applied to the representations actively maintained in mind can help shed light onto the control mechanisms of WM and how its contents can be flexibly updated, strengthened, and/or discarded when information about their relevance becomes available (Tamber-Rosenau, Esterman, Chiu, \& Yantis, 2011). 
This special issue honors Steve Yantis and his many contributions to the understanding of the mechanisms of the control of attention. In one of his more recent publications, Yantis and collaborators (Tamber-Rosenau et al., 2011) timely warned us that we are still far from fully understanding how the selection of representations in WM takes place and how it relates to the selection of perceptual representations (see also Yantis, 2008). This is not a hopeless state; this gap is being gradually narrowed down, and several experimental paradigms are available for probing the mechanisms of attentional selection in WM (for a review, see Oberauer \& Hein, 2012). Here we focus on one of the approaches that has been particularly fruitful in recent years: the retro-cue paradigm.

This review of the retro-cue effect is structured in four sections. In the first section, we describe the retro-cue effect and expand on why this effect has attracted considerable interest in the past 13 years. We then move on to describe the many procedures used to measure retro-cue effects, providing a quick overview of the implementations of the retro-cue paradigm in different visual WM tasks. In the second section, we review five sets of findings characterizing how internal attention works in the retro-cue paradigm. In the third section, we march through six hypotheses that have been proposed to explain the retro-cue effect and evaluate them in light of the available evidence. Finally, we conclude this review with a discussion of potential future directions to inspire developments in the field.

\section{The retro-cue effect}

The retro-cue effect was first described by two independent groups of researchers: Landman, Spekreijse, and Lamme (2003) and Griffin and Nobre (2003). The retro-cue paradigm is in many regards similar to the classical Posner cueing paradigm (Posner, 1980) used to investigate the orienting of attention in the perceptual domain. In the Posner cueing task, a spatial cue guides attention to the location where a relevant stimulus will occur, thereby enhancing detection and processing of the stimulus, as revealed by faster and more accurate responses to the stimulus at the cued location. In the retro-cue paradigm, a spatial cue (called a retro-cue, due to its retroactive or retrospective effect) is shown during the retention interval of a visual WM task. The retro-cue guides attention to one of the representations in WM, thereby tagging it as the most relevant one for an upcoming memory test. ${ }^{1}$ The retrocue correctly indicates the to-be-tested item in an abovechance proportion of trials, referred to as the cue reliability.

\footnotetext{
${ }^{1}$ Typically, retro-cues point to the to-be-tested item; however, it is also possible to use retro-cues to tag irrelevant, to-be-forgotten items (Williams, Hong, Kang, Carlisle, \& Woodman, 2013; Williams \& Woodman, 2012).
}

In line with the cueing advantages observed in perceptual tasks, retro-cues improve performance in tests of the validly cued item, whereas costs are observed for tests of one of the noncued items - henceforth, invalid retro-cue trials (Griffin \& Nobre, 2003; Landman et al., 2003). These effects are not due to modulation of the response to a preferred perceptual stimulus over others (since no perceptual input is present), and no eye movement artifacts (e.g., Matsukura, Luck, \& Vecera, 2007) or eccentricity effects (Tanoue \& Berryhill, 2012) have been found to explain it.

Since its first description in 2003, there has been much research interest in the retro-cue effect. Retro-cues in visual WM paradigms are a versatile tool for making the concept of a focus of attention in WM (Oberauer \& Hein, 2012) empirically tractable. Guiding internal attention through a retro-cue enables researchers to investigate how the information in WM is selected for processing, which information can be selected, how it remains selected, and what consequences attentional selection has for the maintenance of information in WM. Finally, the retro-cue effect indicates that more information can be extracted from WM than what is measured with standard test procedures, hence showing that previously assumed fixed capacity limitations may be due less to the rigid structure of WM and more to how the information in WM is accessed. In a nutshell, the study of the retro-cue effect provides important clues in understanding the structure and functions of WM, and on how capacity limitations can be mitigated by efficiently managing information to match the current task goals.

In the present review, our goal is to synthesize the growing body of research on the retro-cue. Our main focus is on behavioral studies, but we will refer to brain data inasmuch as they help to adjudicate between competing cognitive theories of the retro-cue effect. For the reader interested in the brain profiles associated with directing attention to internal representations in comparison to orienting to perceptual ones, we refer to the recent review by Gazzaley and Nobre (2012).

\section{Measuring the benefits and costs of retro-cues}

The retro-cue has remained, for the most part, an effect investigated in visual WM. Most studies have used simple visual stimuli such as colors (e.g., Griffin \& Nobre, 2003) or oriented bars (Landman et al., 2003), following the footsteps of the authors first demonstrating this effect. However, there are reports of retro-cue effects with more complex visual stimuli, such as alphanumeric or horoscope symbols (Sligte, Scholte, \& Lamme, 2008), shape-color conjunctions (Delvenne, Cleeremans, \& Laloyaux, 2010; Gilchrist, Duarte, \& Verhaeghen, 2016; Li \& Saiki, 2015), colored line-drawing of objects (Astle, Nobre, \& Scerif, 2012; Sligte, Vandenbroucke, Scholte, \& Lamme, 2010), object drawings with 3-D details (Beck \& van Lamsweerde, 2011), real-world pictures of objects (LaRocque et al., 2015), visual illusions 
(Vandenbroucke, Sligte, Fahrenfort, Ambroziak, \& Lamme, 2012), and pictures of faces or scenes (Lepsien \& Nobre, 2007; Lepsien, Thornton, \& Nobre, 2011). There is evidence that retro-cues modulate access to auditory (Backer \& Alain, 2012; Backer, Binns, \& Alain, 2015), tactile (Katus, Andersen, \& Müller, 2012a, b, 2014), and verbal stimuli in WM (Oberauer, 2001, 2002, 2003, 2005). However, given the more prominent role of retro-cues in the visual domain, we will limit this review to studies using visual stimuli.

Retro-cues have been embedded in most of the typical tasks used to measure visual WM: change detection, local recognition, global recognition, continuous delayed estimation, and discrimination of change (see the examples in Fig. 1A). The common feature across all of these tasks is that participants are presented with a memory array containing visual objects. After a brief interval (typically $1 \mathrm{~s}$ ), memory is tested with the presentation of a test display. In most of the tasks, participants are asked to judge whether or not the test display changed in comparison to the memory array. The test display could contain as many probes as the memory array (change detection task; Rensink, 2002) or just one probe presented either in the location of the to-be-judged item (local recognition) or in the center of the display, to be compared to all items in memory (global recognition; Rouder, Morey, Morey, \& Cowan, 2011; Wheeler \& Treisman, 2002).

All of the above-mentioned tasks offer a binary measure of memory performance: The participant responds either correctly (i.e., a hit or correct rejection) or incorrectly (i.e., a miss or a false alarm). Accordingly, accuracy in these tasks has been analyzed using measures such as proportions of correct responses, $d^{\prime}$, or an estimate of capacity $(K)$ derived from hit and false-alarm rates (Rouder et al., 2011). Some studies have also assessed the speed of access to WM's contents, as measured by the reaction times (RTs) on correct trials. For both accuracy (no matter the specific measure) and RTs, retro-cue benefits and costs have been consistently reported (see Gressmann \& Janczyk, 2016, for an overview of benefits and costs).

More recently, tasks have been developed that allow measurement of the fidelity with which an item is remembered in WM (Prinzmetal, Amiri, Allen, \& Edwards, 1998; Wilken \& Ma, 2004; Zhang \& Luck, 2008). In the continuous delayedestimation task, participants are asked to reconstruct the feature of a target item to match the feature stored in memory (see Fig. 1A) using a continuous scale (e.g., a color wheel, or a response dial to rotate an item). The dependent measure in this type of task is response error, measured as the distance between the target item's true feature and the one reported by the participant. The closer the mean response error is to zero, the higher the fidelity of the representation in memory. The distribution of response errors can also be submitted to modeling to extract parameters related to the probability of having recalled the target item (as opposed to guessing or recalling one of the nontarget items) and the variability (precision) with which features from memory are recalled (Bays, Catalao, \& Husain, 2009; Zhang \& Luck, 2008).

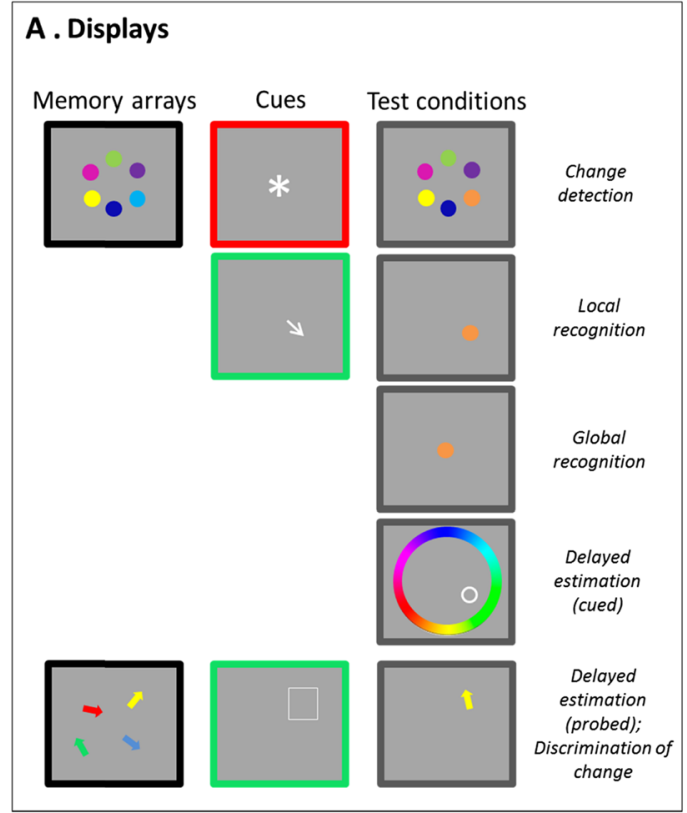

Fig. 1 (A) Different displays (memory arrays, cues, and test conditions) used in visual WM tasks in which retro-cues have been embedded. Memory displays such as those in the first column can be combined with neutral cues (top row) or retro-cues (second and bottom rows), shown in the second column, and tested by one of the five procedures

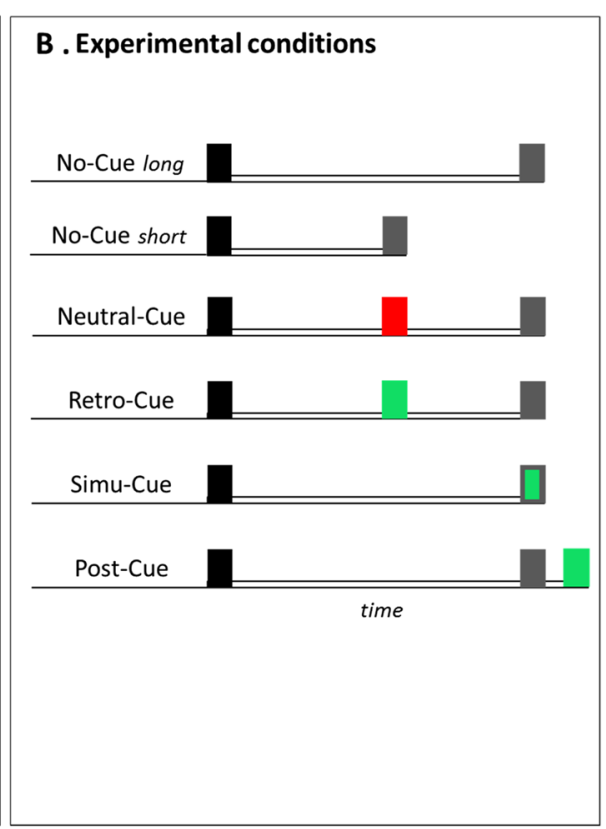

illustrated in the third column. (B) Flows of events in different conditions with and without cues. Black bars represent presentation of the memory array, and gray bars the test display. In the online color figure, informative cues are depicted as green bars, and uninformative (neutral) cues by a red bar 
One final task variant that also offers a measure of WM fidelity is the so-called discrimination-of-change task (Bays \& Husain, 2008). In this task, a test display is shown containing a single probe that is always changed in relation to the corresponding memory item (e.g., a probe arrow displaced $20^{\circ}$ to the left; see Fig. 1A). Participants have to judge in which direction (e.g., left or right) the probe stimulus has changed. The degree of displacement is varied, allowing researchers to measure precision as the slope of the psychometric function relating change identification accuracy to the size of a change, and the probability of reporting information from memory (as opposed to guessing) as the asymptote of the psychometric function at large sizes of change (Bays \& Husain, 2008; Murray, Nobre, Clark, Cravo, \& Stokes, 2013).

The latter two tasks - delayed estimation and change discrimination - have the advantage of allowing researchers to assess which memory parameter, as estimated by a measurement model, is affected by the presentation of a retro-cue (i.e., probability of recall vs. precision). So far, all studies assessing retro-cue effects in continuous tasks have consistently reported benefits in the probability of retrieving the retro-cued item (Gunseli, van Moorselaar, Meeter, \& Olivers, 2015; Makovski \& Pertzov, 2015; Murray et al., 2013; Souza, manuscript submitted for publication; Souza, Rerko, Lin, \& Oberauer, 2014; Souza, Rerko, \& Oberauer, 2016; van Moorselaar, Gunseli, Theeuwes, \& Olivers, 2015; Wallis, Stokes, Cousijn, Woolrich, \& Nobre, 2015; Williams, Hong, Kang, Carlisle, \& Woodman, 2013). Regarding the precision of recall as measured by the mixture model, the findings are mixed. Only about half of the above-mentioned studies observed benefits in this parameter (Gunseli et al., 2015; Makovski \& Pertzov, 2015; Souza, manuscript submitted for publication; van Moorselaar, Gunseli, et al., 2015; Wallis et al., 2015; Williams et al., 2013). At present, it is not clear why some studies find effects on precision, whereas others don't. One pattern that seems to emerge from these studies is that effects on precision tend to appear when memory load is low (i.e., less than four items). ${ }^{2}$

The typical implementation of a retro-cue trial in a WM task comprises presentation during the retention interval of a central arrow cue or a peripheral spatial cue (see the examples in Fig. 1A). Central and peripheral cues are equally effective in producing retro-cue benefits (Matsukura, Cosman, Zachary, Vatterott, \& Vecera, 2014). Despite the ubiquitous use of spatial retro-cues in the literature, there is more to the retro-cue effect than spatial selection. Some studies have used color or shape retro-cues (Heuer \& Schubö, 2016; Li \& Saiki, 2015; Pertzov, Bays, Joseph, \& Husain, 2013), hence engaging

\footnotetext{
${ }^{2}$ One reason for this may be due to unreliable estimation of the memory precision parameter in the mixture model under the low levels of performance (high guessing rate, low precision, or both) that are usually observed in high-memory-load conditions (Lawrence, 2010).
}

feature-based attention to the cued item(s). There is also evidence of effective selection using semantic cues (Backer et al., 2015), or even verbal retro-cues (Gilchrist et al., 2016; Hollingworth \& Maxcey-Richard, 2013).

Typically, retro-cues are presented after a retention interval of at least $0.5 \mathrm{~s}$, to guarantee that the cue is operating outside of the time range of iconic memory (Irwin \& Thomas, 2008; Sperling, 1960). Retro-cue benefits have been consistently reported when the cue appears between 1 and $5.5 \mathrm{~s}$ (Sligte et al., 2008) and up to $9.6 \mathrm{~s}$ (Astle, Nobre, \& Scerif, 2012) after memory array offset, demonstrating that they cannot be reduced to selective readout from iconic memory.

Retro-cue trials have been compared to different baselines, as is shown in Fig. 1B. ${ }^{3}$ The first possibility is to use no-cue trials. No-cue trials can be implemented in two ways: Either the total retention interval is matched to the one used in the retro-cue trial (no-cue long), or the retention interval is reduced to match the time until presentation of the retro-cue in retro-cue trials (no-cue short). The latter condition has the advantage of controlling for time-based forgetting up until the presentation of the retro-cue.

Another popular choice is to use neutral-cue trials. Neutral cues are presented at the same point in time as retro-cues, but unlike the latter, these cues are uninformative, hence not guiding the allocation of attention (see the top of Fig. 1A). This baseline is usually preferred in studies measuring neural markers of attentional control (e.g., Griffin \& Nobre, 2003; Kuo, Stokes, \& Nobre, 2012; Lepsien, Griffin, Devlin, \& Nobre, 2005; Lepsien et al., 2011; Myers, Walther, Wallis, Stokes, \& Nobre, 2015; Nobre, Griffin, \& Rao, 2008), because it equates the retro-cue and baseline trials in terms of the responses to perceptual stimulation. Studies that have included both no-cue and neutral-cue trials (Makovski, Sussman, \& Jiang, 2008; Murray et al., 2013) have observed that performance does not differ between these conditions, provided that they are matched regarding overall retention interval (i.e., no-cue long vs. neutral cue).

Retro-cue trials can also be compared to simultaneous-cue trials (simu-cues, for short). Simu-cues are usually used to restrict the decision processes to one candidate probe stimulus in a change detection test array (e.g., Luck \& Vogel, 1997; Wheeler \& Treisman, 2002), or even in local-recognition tasks (Makovski et al., 2008). Simu-cues have sometimes been found to yield performance similar to that in no-cue trials (Luck \& Vogel, 1997; Wheeler \& Treisman, 2002). However, there is some evidence that simu-cues improve performance relative to no-cue trials by reducing the number of comparisons in memory when the stimuli are complex (e.g., Beck \& van Lamsweerde, 2011; Hollingworth, 2003; Makovski et al., 2008). Finally, retro-cue trials have

\footnotetext{
${ }^{3}$ In this review, we are only considering studies that have assessed retrocue effects against a baseline.
} 
occasionally been compared to postcues - that is, cues presented after the presentation of the test display. Similarly to simu-cues, postcues could potentially be used to narrow down the comparison process to the cued item in change detection, and hence may yield some improvement in comparison to nocue trials (Beck \& van Lamsweerde, 2011; Landman, Spekreijse, \& Lamme, 2004).

\section{The empirical characteristics of retro-cue effects}

The retro-cue effect is thought to arise due to the allocation of attention to the cued item. In this section, we briefly review five lines of investigation speaking to how attention is guided by a retro-cue.

\section{Time course}

How much time does one need to make effective use of a retrocue? A few studies have addressed this question by varying the temporal separation between the retro-cue and onset of the test display (hereafter, the cue-test delay). Tanoue and Berryhill (2012) varied the cue-test delay from 100 to $700 \mathrm{~ms}$ in a local-recognition task. Significant retro-cue benefits emerged after $300 \mathrm{~ms}$, and they did not increase in size with longer cue-test delays. Souza, Rerko, and Oberauer (2014) also varied the cue-test delay $(100,400,1,000$, or 2,000 ms) in local recognition (see also Gressmann \& Janczyk, 2016). Retro-cue benefits for accuracy were stable after a delay of $400 \mathrm{~ms}$, but benefits for RTs continued to increase up to $1,000 \mathrm{~ms}$. Studies varying cue-test delay in delayed-estimation tasks (Pertzov et al., 2013; Souza et al., 2016) have also shown retro-cue effects for delays of $300 \mathrm{~ms}$ or more. ${ }^{4}$ Some studies have also assessed how long it takes to achieve a stable retro-cue benefit by varying the stimulus onset asynchrony (SOA) between the offset of the retro-cue and presentation of a visual mask (Pinto, Sligte, Shapiro, \& Lamme, 2013; van Moorselaar, Gunseli, et al., 2015). The visual mask diminished the retrocue benefit with SOAs up to $300 \mathrm{~ms}$, further supporting this interval as the time needed to observe the full retro-cue benefit.

In sum, people need at least $300-500 \mathrm{~ms}$ to make full use of the retro-cue to improve the accuracy of their responses, with performance remaining stable thereafter. The speed of processing, however, can continue to improve up to $1,000 \mathrm{~ms}$ after the retro-cue.

\footnotetext{
${ }^{4}$ In Pertzov et al. (2013), retro-cue benefits in recall error continued to increase with longer cue-test delays. It may be worth noting, however, that the overall retention interval in the baseline condition was also lengthened to match the total duration of the retro-cue trial (no-cue long condition). Hence, this effect may have been due to increased time-based forgetting (due to decay or interference) in the baseline.
}

\section{Voluntary/strategic control}

A second question concerns the voluntary control of the allocation of attention to the retro-cue. Some studies have investigated whether the retro-cue triggers an automatic shift of attention to the cued location or whether this shift is voluntary and dependent on the informational value of the cue - a distinction much like that between stimulus-driven versus goaldirected perceptual attention (Yantis, 2000). The degree of voluntary control has been measured through manipulations of cue reliability - that is, the proportion of trials on which the cue validly indicates the test item. ${ }^{5}$

Gunseli, van Moorselaar, Meeter, and Olivers (2015) compared retro-cue benefits and costs in an orientation delayedestimation task with cue reliabilities of $50 \%$ and $80 \%$ (random selection of the target of recall would yield a $25 \%$ reliability). In both conditions, retro-cue benefits were observed, but these benefits were larger under the higher-reliability condition. Invalid retro-cue costs, conversely, showed up only in the high-reliability condition. These results seem to indicate that people use the retro-cue differently on the basis of how predictive it is of the to-be-tested item. Berryhill, Richmond, Shay, and Olson (2012) compared 100\%-reliable to completely uninformative retro-cues. Presenting a retro-cue improved testing of the retro-cued item even when this cue was uninformative, but the retro-cue effect was smaller in the nonpredictive condition. ${ }^{6}$ Shimi, Nobre, Astle, and Scerif (2014) compared the performances of different groups of participants exposed to retro-cues with $100 \%$ or $50 \%$ reliability. Retro-cue benefits were reduced under the low-reliability condition, being only significant when compared against invalid-cue trials. In sum, retro-cue effects (benefits and costs) depend on the reliability of the retro-cue, indicating that people use it strategically to modulate WM maintenance. When retro-cues are mostly valid, noncued items become irrelevant and may be worth discarding to reduce memory load, even though that incurs a performance cost on the rare invalid-cue trials. When retro-cues are informative but have low reliability, removing noncued items is not a good strategy, but attention can still be used to strengthen the retro-cued item, thereby generating a benefit for valid-cue trials with little cost for invalid-cue trials (Gunseli et al., 2015).

\section{Resistance to distraction}

A third line of investigation has assessed whether attention needs to remain focused on the retro-cued item until test to obtain a retro-cue benefit. Hollingworth and Maxcey-Richard

\footnotetext{
${ }^{5}$ The proportion of valid cues is often referred to as the cue validityhere we use the term validity to characterize whether an individual cue points to the target (i.e., a valid cue) or not (i.e., an invalid cue), and use reliability for the overall proportion of valid cues.

${ }^{6}$ These authors did not present an analysis of retro-cue costs for invalid-cue trials.
} 
(2013) tested whether the retro-cue requires sustained spatial visual attention to the cued item by introducing, in some trials, a visual search task after presentation of either a neutral cue or a retro-cue. The visual search task was meant to prevent spatial attention from being persistently focused on the retro-cued item. Although the visual search task impaired memory, it did not reduce the retro-cue benefit. Rerko, Souza, and Oberauer (2014) applied a similar logic to test the contribution of feature-based attention. The interruption task in this study required participants to make a perceptual judgment (warmcold color decision) on a centrally displayed stimulus during the retention interval (presented after the retro-cue in retro-cue trials). Again, the interruption task impaired overall memory performance but did not reduce the retro-cue benefit. In a second step, Rerko et al. also tested whether the retro-cue benefit is robust against distraction from the focus of attention within WM: After retro-cueing an item A, participants were first tested on another item $B$ before being tested on the cued item A. Because retrieving item $B$ required selecting $B$ at the exclusion of all other items in the memory set, the focus of attention could not remain on A continuously until it was tested. Yet the retro-cue benefit for item A was undiminished by the intervening retrieval of another item from WM.

The maintenance of retro-cue benefits after distraction from the focus of attention has also been observed in the delayedestimation task. Makovski and Pertzov (2015) inserted a parity decision on visually or auditorily presented digits after the presentation of a cue (retro-cue or neutral cue). The parity task (in both visual and auditory forms) impaired performance in both cue conditions, but less so in the retro-cue condition, consistent with the idea that the retro-cueing benefit survives distraction of central attention.

This conclusion is further supported by a study measuring the neural substrate of attention after presentation of a retrocue. Wallis et al. (2015) tracked alpha-power lateralization, which is thought to index the deployment of attention, during the time following a retro-cue. They observed that alpha lateralization occurs only transiently (for about $500 \mathrm{~ms}$ ) after the retro-cue, suggesting that attention does not persistently linger on the retro-cued item, even when its focus is not distracted by a secondary task. These findings also converge with the time course of obtaining a retro-cue benefit, which seems to require no more than $500 \mathrm{~ms}$.

In a related vein, Zokaei, Ning, Manohar, Feredoes, and Husain (2014) investigated whether the maintenance of a retro-cued item could be disrupted by the application of transcranial magnetic stimulation (TMS) to areas involved in the sensory perception of the stimulus that is currently maintained in WM. Zokaei et al. asked participants to maintain the motion direction of two dot patterns. After presenting a retro-cue, TMS was applied to motion-sensitive area MT+. The TMS impaired performance overall, but the retro-cue benefit was not reduced by it. This finding contrasts with the observation that stimulation of MT+ has been found to disrupt other effects that have been thought to reflect attention in WM, such as the recency effect and an incidental cueing benefit (Zokaei, Manohar, Husain, \& Feredoes, 2014).

Taken together, the results above establish that the retrocue benefit is robust against distraction of spatial or featurebased attention, shifts of attention within WM, and disruption of maintenance through TMS over stimulus-relevant sensory areas. These findings converge with other studies that have observed that one item can be retained in a privileged state (of heightened accessibility) even when other items are cued between the initial cueing and testing (Rerko \& Oberauer, 2013; Souza, Rerko, \& Oberauer, 2015) or are subsequently encoded into WM (Gorgoraptis, Catalao, Bays, \& Husain, 2011; Maxcey-Richard \& Hollingworth, 2013; Souza, Rerko, \& Oberauer, 2014). In sum, focused attention can be used to prioritize one WM item in a manner that is robust against distractions from the external or internal focus of attention.

\section{Central attention demands}

Some recent evidence has been provided to bear on the question of the attentional demands of using the retro-cue. Janczyk and Berryhill (2014) assessed whether there is a critical period in which central attention to the retro-cued item is required, by measuring dual-task interference using the psychological refractory period (PRP) paradigm. In the absence of perceptual and motor overlap between the two tasks, dual-task costs in the PRP paradigm reflect competition for central attention, conceptualized as a general processing bottleneck (Pashler, 1994) or resource allocated (Tombu \& Jolicœur, 2003) for processes such as response selection and retrieval from longterm memory. Janczyk and Berryhill inserted a tone discrimination task during the retention interval of a local-recognition task and varied the temporal separation (SOA) between presentation of the tone and subsequent presentation of a cue (retro-cue or neutral-cue). If central attention is required temporarily for using the retro-cue, then short SOAs should create a situation of competition for central attention, leading to smaller retro-cue benefits than would be observed at longer SOAs. This is exactly what they observed when comparing SOAs of 150 and $850 \mathrm{~ms}$, showing therefore that central attention is required over a critical period to make use of the retro-cue.

\section{Splitting attention between multiple WM items}

Retro-cues can be used to guide attention to one single item or to multiple items in WM. To date, there is still dispute whether simultaneously retro-cueing multiple items (hence, requiring concurrent selection of several representations in WM) leads to an advantage for memory performance relative to a no-cue 
baseline. Makovski and Jiang (2007) compared the efficacy of one-cue, two-cue, and three-cue displays in yielding a retrocue benefit. Only single retro-cues improved performance in their study. Delvenne and Holt (2012) showed evidence of two-cue benefits, provided that the cues pointed to different items across left-right hemifields. ${ }^{7}$ Other studies have used cues pointing to entire memory sets (set cues), such as cues indicating all of the items on the left or the right side of the screen as being relevant (Matsukura et al., 2007; Williams \& Woodman, 2012), which were successful in improving performance. Recently, Matsukura and Vecera (2015) tested the boundary conditions for observing benefits yielded by simultaneously retro-cueing multiple items. These authors compared single cues, muti-item cues (cueing a random subset of three out of six items), and set cues (cueing groups of three contiguous items). Critically, in this study, the testing procedure was varied, testing a single item from the retro-cued set (i.e., a local-recognition test) or testing the entire set of retrocued items (i.e., a change detection test on the cued subset of the array). Multi-item cues and set cues only benefited performance when the entire set was tested, suggesting that the test display has to match the attentional set created by the retrocue. Yet another recent study conducted by Heuer and Schubö (2016) has opened up more possible alternative explanations for these inconsistencies. In their study, they cued two items from a memory array simultaneously using either spatial cues (arrows), symbolic cues (numbers mapped to locations), or feature-based cues (color or shape). Moreover, they also varied the spatial distance between the cued items, such that the items could be from neighboring or nonneighboring positions. Spatial and symbolic cues only improved performance relative to a neutral-cue condition when neighboring locations were cued. In contrast, feature-based cues yielded benefits, irrespective of the spatial distance between cued items. These findings suggest that the selection of multiple items simultaneously may be contingent on how different forms of attention (spatial or feature-based) are engaged by the retro-cue.

Whereas the use of simultaneous multi-item cues has yielded a mixed pattern, sequential presentation of multiple retro-cues has shown that participants can shift attention between items in WM. When a second retro-cue follows the first, and the last-cued item is the one relevant to the test, a retro-cue benefit is observed for the second-cued item (Landman et al., 2003; Li \& Saiki, 2014; Maxcey, Fukuda, Song, \& Woodman, 2015; Rerko \& Oberauer, 2013). When all of the sequentially cued items are relevant, improvements have been reported for all cued items (Li \& Saiki, 2014; Souza et al., 2015), and this improvement is larger, the more

\footnotetext{
${ }^{7}$ The findings of Delvenne and Holt (2012), however, stand in contrast to those reported by Makovski and Jiang (2007) because in the latter study, the two cues always pointed to items in different hemifields, with no benefits being observed.
}

frequently the same item is cued during the retention interval (Souza et al., 2015). There is only one exception to this pattern, revealed in a study by van Moorselaar, Olivers, Theeuwes, Lamme, and Sligte (2015). In this study, a second retro-cue did not bring performance above baseline, but it did counteract the costs otherwise incurred by an invalid first retro-cue. One factor that may explain these discrepant results is the frequency of two-cue trials, which were much less frequent than single-cue trials in van Moorselaar, Olivers, et al. These results therefore suggest that the strategic use of retro-cues is not only sensitive to cue reliability, but also to the frequency with which a first cue is followed by a second one.

\section{Interim summary}

In a nutshell, the studies reviewed above show that the retrocue induces a voluntary shift of (central) attention to the cued item for about $300-500 \mathrm{~ms}$. After this period, the retro-cued item reaches a robust, privileged state in WM. This state involves more than remaining selected within the focus of attention, because distracting attention from the cued object by requiring the selection of a response to a perceptual stimulus, or selection of another item within WM, does not diminish the retro-cue benefit.

The emergent picture is that a state of robustness can be achieved for multiple items in WM, provided that they are focused on sequentially. Focusing of multiple items simultaneously may also provide a benefit, but the boundary conditions for that effect are not yet firmly established.

\section{Explanations of the retro-cue effect}

The retro-cue effect is puzzling to explain from a pure informational standpoint. Consider a task in which a single item is tested (e.g., in local recognition or change discrimination). In the retro-cue condition, a retro-cue at time $t_{1}$ only provides one piece of information: It indicates which item will be tested. All further information is delayed until the test display is presented at a later time $t_{2}$. In contrast, in a no-cue short condition (see Fig. 1), the memory test instead occurs at time $t_{1}$. The test display contains more information than the retro-cue: It identifies which item is tested, provides the probe to compare that item to, and allows decision making to start. Yet, robust retrocue benefits have been found in comparison to a no-cue short condition (Makovski et al., 2008; Murray et al., 2013; Rerko et al., 2014; Souza, Rerko, Lin, et al., 2014; Souza et al., 2015; Souza, Rerko, \& Oberauer, 2014). How can less information be helpful? Intriguingly, people can use the retro-cue to access and modulate WM contents more effectively than 
when they are given a probe that includes more information than the retro-cue.

In this section, we review six hypotheses about the causes of the retro-cue effect that have featured in the literature. Our goal is to make explicit their main assumptions and predictions, and to review how they have been empirically tested so far. Table 1 provides a brief overview of the six retro-cue hypotheses described herein. These hypotheses are not mutually exclusive. Therefore, evidence corroborating one hypothesized cause of retro-cue effects does not rule out the contribution of another cause.

Table 1 Explanations of the retro-cue benefit

Hypothesis

Assumptions and Predictions

H1. Protection from time-based decay

Item in visual WM suffer from time-based decay. Decay affects all items in memory unless some active mechanism is used to counteract forgetting.

A retro-cued item is protected from time-based decay, whereas noncued items are left unprotected from time-based decay.

H2. Prioritization for comparison

At test, items are serially searched in memory to be compared to the probe stimulus. Each comparison step is time-consuming and error-prone. The more comparisons that are made to reach a decision, the slower and more error-prone the response is.

The retro-cue reduces the number of comparisons made in memory, yielding faster and more accurate responses.

H3. Removal

Memory items interfere with each other (interitem competition), as reflected in the set-size effect.

The retro-cue indicates which information is no longer relevant and can therefore be removed (discarded) from WM.

Removal reduces memory load, freeing capacity to process the relevant (cued) information and to add new input to WM.

H4. Attentional strengthening or refreshing

Attention is used to augment the accessibility of representations in WM (refresh its trace). Arguably, in no-cue trials refreshing is distributed among all items in memory.

The retro-cue indicates which item can be selectively attended to, and thereby differentially strengthened in memory.

H5. Retrieval head start

Items have to be retrieved (selected) from working memory for making a decision. Retrieval can be understood as the gradual accumulation of evidence for an item in memory.

The retro-cue allows evidence to accumulate for the target item ahead of decision making (i.e., response selection), increasing the probability that the correct information will be selected for decision making.

H6. Protection from perceptual interference

Visual input (e.g., a visual mask, the probe stimulus, or a color wheel) following encoding of the memory array can replace or distort memory representations.

The retro-cued item is insulated from interference by subsequent visual input.

\section{H1. Protection from time-based decay}

This hypothesis rests on the assumption that representations in visual WM suffer from time-based decay, and that decay can be (partially) counteracted by attending to representations. When a retro-cue is presented, attention no longer has to be distributed among all items, but can be devoted exclusively to the retro-cued item, protecting it from further forgetting (see Fig. 2A). Testing of a noncued item in invalidcue trials should lead to progressively larger costs over longer postcue intervals that these items are unprotected from decay. This pattern is exactly what was obtained by Pertzov et al. (2013) using a continuous color and continuous orientation delayed-estimation task. In contrast, Gressmann and Janczyk (2016) did not find evidence matching these predictions in a local-recognition task. In this study, memory arrays with four or eight colors were followed by neutral cues and retro-cues ( $80 \%$ valid). The cue-test delay was varied from 100 to $1,900 \mathrm{~ms}$. Valid retro-cue benefits and invalid retro-cue costs were observed as compared to the neutral-cue condition after a cue-test delay of $400 \mathrm{~ms}$, and remained stable thereafter. Note that this study also did not find any worsening of performance over longer cue-test delays in the neutral-cue (baseline) condition-hence, not showing any decay of memory representations. Similar findings were also reported by van Moorselaar, Olivers, et al. (2015): no worsening of performance with long and short no-cue baselines, and no increase of retro-cue costs with longer cue-test delays.

Protection from decay could arguably explain the retrocue benefit in comparison to a baseline condition that was matched in terms of overall retention interval (i.e., the nocue long or neutral-cue conditions in Fig. 1). However, protection from decay cannot explain retro-cue benefits in comparison to no-cue short trials, because in that comparison there is as much time for decay before the retro-cue as in the control condition. In Fig. 2A, this is illustrated by the common decay curve of all conditions up to the onset of the cue, which coincides with the onset of the test probe in the no-cue short condition. Makovski et al. (2008) were the first to demonstrate that retro-cue benefits are observed relative to a no-cue short baseline. This finding has since been replicated in several other reports (Murray et al., 2013; Rerko et al., 2014; Souza, Rerko, Lin, \& Oberauer, 2014; Souza et al., 2014, 2015). These studies show that there is more to the retro-cue benefit than protection from time-based decay.

Whether time-based decay contributes to forgetting from visual WM is in itself still a matter of debate. In visual WM tests, performance has been found to be inversely related to the duration of the retention interval (Morey \& Bieler, 2013; Pertzov et al., 2013; Ricker \& Cowan, 2010, 2014; Ricker, Spiegel, \& Cowan, 2014; Shipstead \& Engle, 2013; Souza \& 

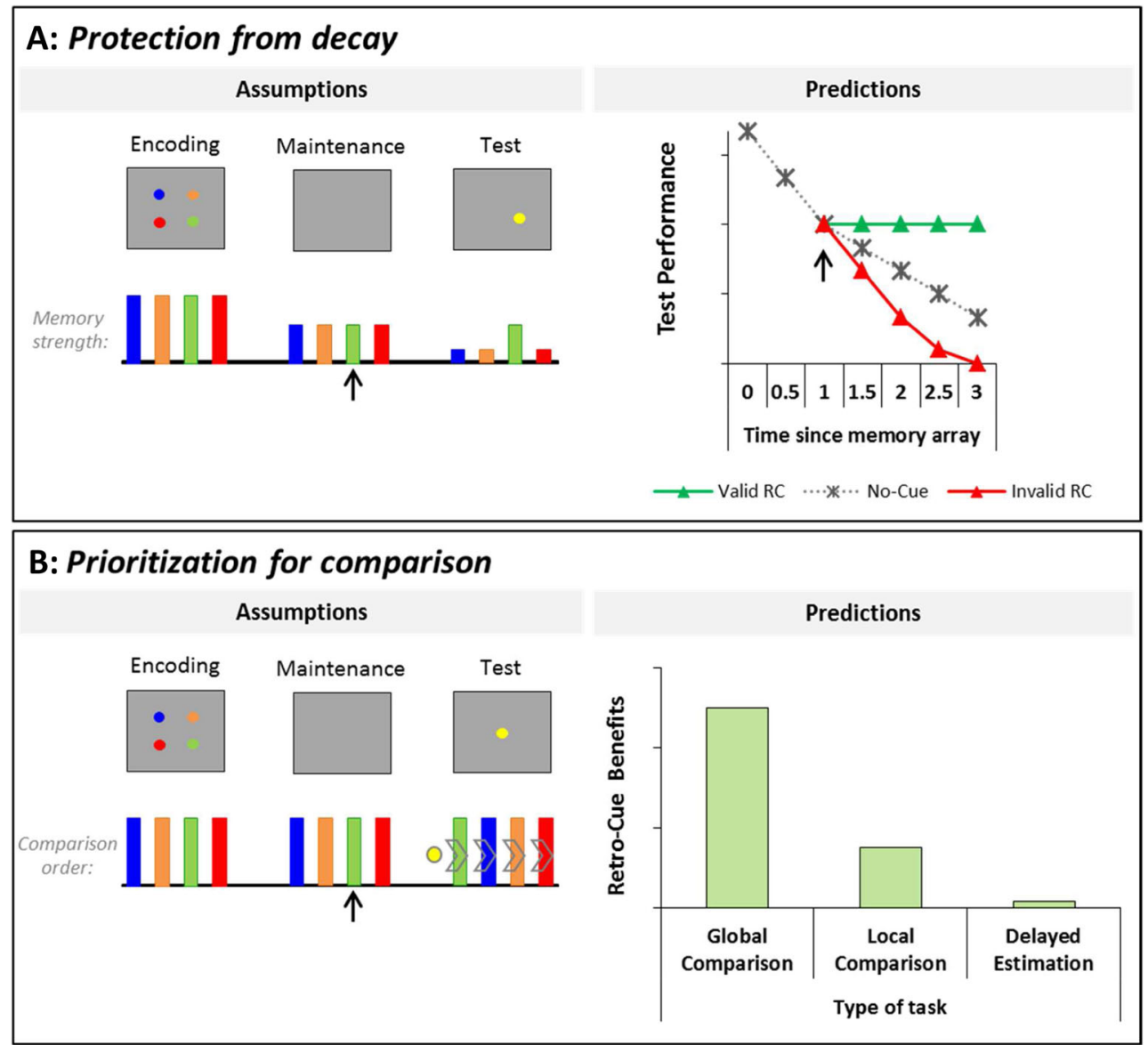

Fig. 2 Assumptions and predictions of retro-cue hypotheses. (A) Protection from decay: The strength of representations in WM decreases over time, leading to forgetting. Focused attention reduces the rate or probability of forgetting for the retro-cued item. This entails that the representation of the retro-cued item remains stable over time, whereas the performance in no-cue and invalid retro-cue trials deteriorates. (B) Prioritization for comparison: At test, memory is searched serially, the the retro-cue changes the order in which items are searched in WM. This entails that retro-cue benefits should be less likely to emerge in tasks in which only one comparison is required (e.g., local recognition), and should be absent in tasks requiring no comparison at all (e.g., delayed estimation). $\mathrm{RC}=$ retro-cue
Oberauer, 2015; Zhang \& Luck, 2009). This is line with a decay explanation of forgetting, but also with an explanation by temporal distinctiveness: As the memory stimuli of the current trial recede into the past, they become less distinguishable in time from preceding trials. An effect of temporal distinctiveness is predicted by interference theories assuming that temporal context is a retrieval cue, such that events close in psychological time compete with each other for retrieval, leading to interference (Brown, Neath, \& Chater, 2007; Brown, Sala, Foster, \& Vousden, 2007). There is experimental support for an interference explanation of time-based forgetting in visual WM (Mercer, 2014; Shipstead \& Engle, 2013; Souza $\&$ Oberauer, 2015; but see Ricker et al., 2014, for conflicting results).

In sum, protection from decay is a noncompelling explanation of retro-cue benefits for three reasons: (1) Retro-cue benefits are observed even in conditions that control for the contribution of time-based decay (no-cue short); (2) there is no consistent evidence for progressive forgetting of noncued items, as would be predicted if these items were unprotected from decay; and (3) the power of time-based decay to explain forgetting in visual WM has been questioned by several recent studies demonstrating the viability of an alternative explanation in terms of time-based interference.

\section{H2. Prioritization for comparison}

This account starts from the assumption that multiple items in visual WM must be searched through sequentially at test. When an item is retro-cued, it receives priority in the search sequence, as is shown in Fig. 2B (Astle, Summerfield, Griffin, \& Nobre, 2012; Makovski et al., 2008; Matsukura et al., 2007; Pertzov et al., 2013). Under the reasonable assumption that each comparison step in memory is a time-consuming and error-prone process, this hypothesis predicts benefits in RTs and accuracy when the number of memory comparisons is reduced. According to this hypothesis, the retro-cue benefit is a function of how much participants rely on a serial search 
through memory to arrive at a response. It is conceivable that participants use serial search in the classical change detection task, in which every stimulus in the test display has to be compared to its counterpart in the remembered memory array, or in a global-recognition task, in which a single, central probe stimulus must be compared to all memory items. However, prioritization is a less plausible explanation when the task clearly limits the comparison process to a single item, as in a local-recognition task, in which the probe has to be compared only to the item presented in the same location. A prioritization account is even less plausible in delayed-estimation tasks, in which a single item is reconstructed from memory. Unlike the predictions shown in Fig. 2B, retro-cue benefits have been shown with all five types of WM tests shown in Fig. 1, rendering the prioritization account nonappealing.

\section{H3. Removal}

When a retro-cue reliably indicates the to-be-tested item, it also tags the other, noncued items as being irrelevant to the memory test. In this scenario, it would be advantageous for participants to get rid of the noncued items, thereby freeing WM capacity to process the cued item and/or encode new relevant information. The active process of getting rid of irrelevant information from WM has been termed removal (Oberauer, 2001). A hallmark of removal is that WM capacity is freed up (see Fig. 3A). This translates into three predictions: (1) The retro-cue benefit occurs at the expense of noncued items, incurring costs on invalid retro-cue trials; (2) the behavioral and neural markers associated with increased memory load (so-called set-size effects) are attenuated as the noncued items are removed from WM, leading to increasingly larger retro-cue benefits as more items are removed from WM; and (3) the more items are removed, the more capacity is freed up to be used to encode new items to WM. Whereas the first two predictions can also be made on the basis of other hypotheses about the nature of the retro-cue effect, the third prediction is unique to the removal hypothesis.

Williams, Hong, Kang, Carlisle, and Woodman (2013) provided strong evidence for the first prediction. Participants encoded two items for a delayed-estimation test, and in half of the trials, one item was retro-cued as irrelevant. Performance on tests of the remaining, relevant item improved in retro-cue trials relative to no-cue trials, and a surprise test of the cued to-beforgotten item showed virtually no memory for that item. Furthermore, several studies have shown invalid retro-cueing costs, which are consistent with a role of removal as an explanation of the retro-cue benefit (Astle, Summerfield, et al., 2012; Gözenman, Tanoue, Metoyer, \& Berryhill, 2014; Gressmann \& Janczyk, 2016; Griffin \& Nobre, 2003; Gunseli et al., 2015; Pertzov et al., 2013; van Moorselaar, Olivers, et al., 2015).

Souza et al. (2014) tested Predictions 2 and 3 in a localrecognition task. They presented no-cue trials or $100 \%$-valid retro-cues, and varied memory load (two, four, or six items). The performance in no-cue trials decreased with memory load. The retro-cue led to a larger improvement of performance at larger memory loads, though it did not entirely eliminate the effect of memory load on accuracy. Several other studies have also revealed larger retro-cue benefits with increasing numbers of noncued items (Gilchrist et al., 2016; Gressmann \& Janczyk, 2016; Kuo et al., 2012; Nobre et al., 2008; Sligte et al., 2008; Souza, Rerko, Lin, \& Oberauer, 2014; Vandenbroucke, Sligte, de Vries, Cohen, \& Lamme, 2015; van Moorselaar, Olivers, et al., 2015), but it is worth noting three studies that have not observed such an interaction of retro-cueing and memory load (Makovski et al., 2008; Matsukura et al., 2007; Trapp \& Lepsien, 2012).

To test whether this interaction indeed reflected freeing of WM capacity after a retro-cue, Souza et al. (2014) asked participants to encode two sequentially presented memory arrays separated by a 2 -s interset interval. During the interset interval, an item from Array 1 was retro-cued in half of the trials. The usual retro-cue benefit was obtained in tests of Array 1 . Critically, testing memory for Array 2 also showed improved performance after a retro-cue to an item in Array 1, showing that the retro-cue freed capacity to encode Array 2 into WM.

A contribution of removal to the retro-cue effect has also been supported by neural evidence. Kuo et al. (2012) assessed how the contralateral delay activity (CDA; a lateralized electroencephalography [EEG] marker of WM load) is modulated by neutral versus retro-cues. In trials without retro-cues, the CDA was larger when participants maintained four items instead of two items in WM. After retro-cueing, however, the CDA was reduced and converged to similar levels for both set sizes, suggesting that only the cued item was maintained in WM (see also Duarte et al., 2013; Schneider, Mertes, \& Wascher, 2015). Another set of studies assessed the neural markers of WM maintenance by using pattern classifiers trained to identify the class of stimuli (words, orientations, etc.) that a person was holding in WM from their brain activity as measured by means of fMRI or EEG. When people hold stimuli from two different classes in mind and one class of stimuli is subsequently retro-cued, the neural activity pattern reflecting the noncued stimulus class drops to baseline, whereas the activity related to the cued class remains measurable (LaRocque, Lewis-Peacock, Drysdale, Oberauer, \& Postle, 2013; Lewis-Peacock, Drysdale, Oberauer, \& Postle, 2012).

These studies show that noncued items are removed from the set of representations maintained through persistent neural activity. At the same time, these items are not irreversibly lost - when initially noncued items are subsequently cued as being relevant, the neural activity pattern reflecting their stimulus class reemerges, and participants can make accurate judgments on these items (LaRocque et al., 2013; Lewis-Peacock 


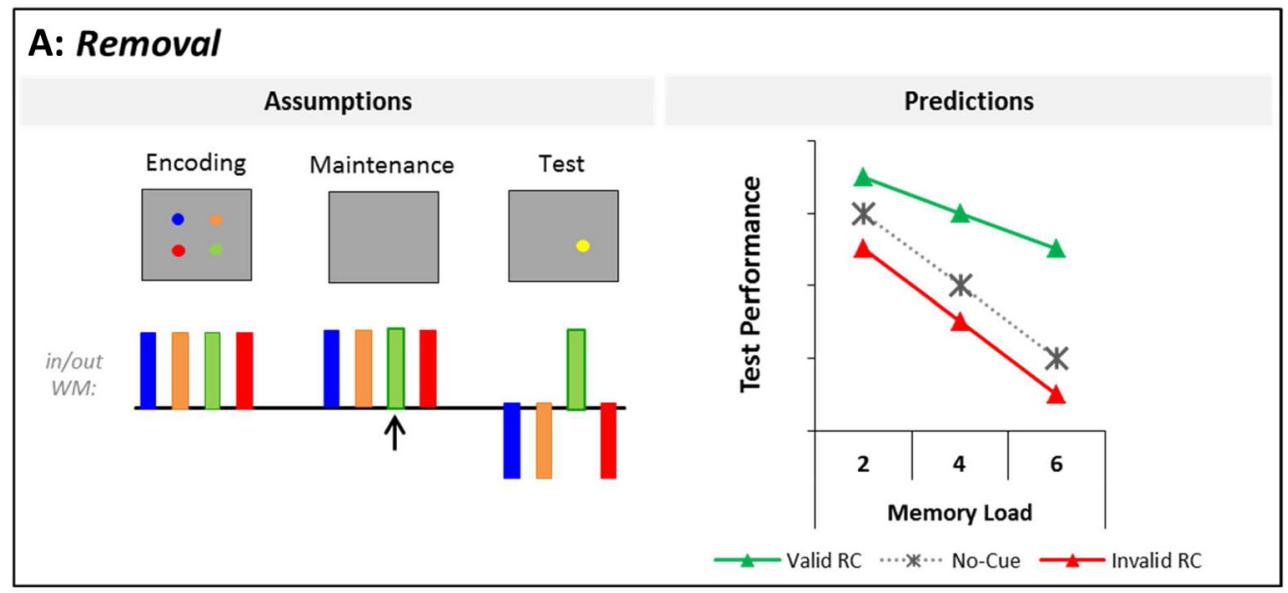

B: Attentional strengthening or refreshing

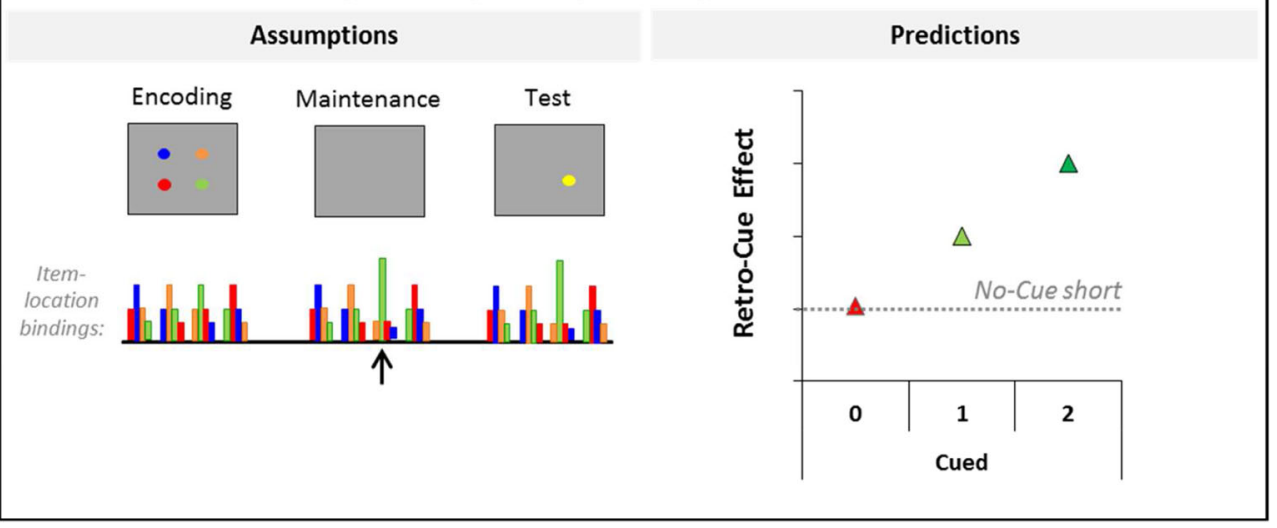

Fig. 3 Assumptions and predictions of retro-cue hypotheses. (A) Removal: Noncued information is rendered irrelevant by a retro-cue. This information is actively removed from WM, freeing capacity. This entails the prediction of increasing benefits, the larger the number of noncued items. Removal also yields costs for tests of the noncued

et al., 2012). Information about these items is apparently maintained in an "activity-silent" state (Stokes, 2015). It is plausible that outsourcing representations to an activitysilent form of maintenance reduces the degree to which they interfere with access to the cued items. When tested directly, the information maintained in activity-silent form might be less accessible than information maintained through persistent neural activity, resulting in an invalid-cue cost. Yet, activitysilent information can apparently be partially (van Moorselaar, Olivers, et al., 2015) or fully (Landman et al., 2003; Rerko \& Oberauer, 2013) recovered when the initially noncued items are cued by a second retro-cue before being tested.

To conclude, there is compelling evidence that the removal of noncued information from WM contributes to the retro-cue benefit. It is unlikely, however, that removal alone suffices for a complete explanation of the retro-cue benefit, because such benefits have been observed also at low levels of cue reliability, such that the removal of noncued items is not advantageous, and experiments have shown no cost of accessing an invalidly cued item under these conditions (Gunseli et al., 2015). items. (B) Attentional strengthening or refreshing: Item-context bindings are strengthened by attention, leading to quick, easy retrieval of the retro-cued item. This entails that noncued items are maintained unchanged in WM, whereas focused (cued) items are incrementally augmented in $\mathrm{WM}$. $\mathrm{RC}=$ retro-cue

\section{H4. Attentional strengthening or refreshing}

Bringing an item to the focus of attention is assumed to strengthen its trace in WM, improving its accessibility for later use, a process often referred to as refreshing (Chun \& Johnson, 2011; Johnson, 1992; Rerko \& Oberauer, 2013; Ricker \& Cowan, 2010; Souza et al., 2015). This hypothesis states that focused attention makes the representation of the retro-cued item, and possibly the binding to its context (e.g., its spatial location), stronger than it originally was after encoding, as is illustrated in Fig. 3B (Kuo, Yeh, Chen, \& D’Esposito, 2011; Lepsien et al., 2011; Nobre et al., 2008; Rerko \& Oberauer, 2013; Rerko et al., 2014; Souza et al., 2014, 2015).

The strengthening of items and of item-context bindings can be distinguished in the local-recognition paradigm (see Fig. 1), by using intrusion probes. These probes test an item $\mathrm{B}$ with a probe matching a different item A from the current memory set. If a retro-cue to item A strengthens the representation of that item, then 
A should be a very strong lure, leading to increased false-alarm rates as compared to an intrusion probe matching a noncued item $C$. In contrast, if a retro-cue to A strengthens the bindings between $\mathrm{A}$ and its location, people should find it easier to correctly reject an intrusion probe matching $\mathrm{A}$ than an intrusion probe matching $\mathrm{C}$. The latter pattern was observed by Rerko and Oberauer (2013), implying that a retro-cue results in stronger item-context bindings, not stronger item representations independent of their context.

A distinguishing feature of the strengthening or refreshing explanation is that noncued items are assumed to be maintained in WM unchanged, whereas the focused item increases in accessibility (Rerko \& Oberauer, 2013). Strengthening does not mean to shift memory strength away from other items and toward the cued item; rather, attending to an item in WM leads to a stronger binding of that item to its context, without affecting the strength of other item-context bindings.

One prediction of this hypothesis is that a retro-cue benefit for validly cued items may occur without a cost for invalidly cued items. Several studies have demonstrated costs of invalid retro-cues (e.g., Astle, Summerfield, et al., 2012; Griffin \& Nobre, 2003; Pertzov et al., 2013). Retro-cue costs are observed even when participants are aware that one of the noncued items will also be tested (Rerko et al., 2014). However, these studies compared invalid retro-cue trials against a no-cue long or a neutral-cue baseline. In the baseline condition, participants probably distributed their attention among several items, refreshing each of them to a small extent, whereas in the retro-cue condition they concentrated attention more exclusively on the cued item. Therefore, the observed costs may not reflect a weakening of the noncued items, but rather a reduction of refreshing opportunities for the noncued items in retro-cue trials as compared to the baseline.

Alternatively, the invalid retro-cue costs may reflect the operation of other processes that, in addition to refreshing, take place in retro-cue trials (e.g., the removal of noncued items, as we explained above). It follows that to observe a pure effect of refreshing (if there is one), one needs to reduce the incentive to remove noncued items from WM. Li and Saiki (2014) have provided a first hint in this direction. In addition to having a retro-cue condition, they presented in some trials a "withdraw cue" after the retro-cue. These cues were used to instruct participants to withdraw attention from the retro-cued item and to reset to a state of maintaining all items equally. Despite presenting this withdraw cue, the probability of testing the retro-cued item or one of the noncued items was maintained the same as in a regular retro-cue trial. In the standard retro-cue trial, testing of validly and invalidly retro-cued items yielded benefits and costs, respectively. In the withdraw condition, valid retro-cue benefits were maintained, but invalid retro-cue costs disappeared. ${ }^{8}$ These results are in line with the idea that participants can strengthen an item without costs for noncued items. Similar findings have been reported from studies that have varied cue reliability: Under low retro-cue reliability conditions, valid retro-cue benefits are still observed, but invalid retro-cue costs disappear (Berryhill et al., 2012; Gunseli et al., 2015; Shimi et al., 2014; Souza et al., 2015).

Another prediction from the strengthening hypothesis is that several items can be focused in succession, leading to an incremental benefit for all of them. Rerko and Oberauer (2013) have provided initial evidence that multiple successive retro-cues do cumulatively strengthen items in WM: When participants see up to three successive retro-cues (with the last-cued item being the one relevant for the test), memory is better for items cued twice (sequence of cueing A-B-A) than for items cued once (sequence $\mathrm{C}-\mathrm{B}-\mathrm{A}$ ). These findings provide a hint that focusing attention on an item in WM strengthens its representation in a cumulative fashion.

Souza et al. (2015) have provided a stronger test of the strengthening explanation. In this study, a sequence of four central arrow cues was embedded in the retention interval of a color delayed-estimation task. Participants were instructed to "think of" (i.e., refresh) the cued items. They were instructed that the cues were nonpredictive of the test item, to reduce any incentive to remove items from WM. Across the sequence of four cues, individual memory items were cued zero, one, or two times. Response errors decreased as the number of refreshing steps directed at the target increased, showing that refreshing benefited memory in a cumulative fashion. Moreover, the zero-cued items in the refreshing condition were recalled as well as items in a no-cue short condition, demonstrating that focused attention not merely protects memory representations from forgetting, but improves memory over time, as is predicted by the strengthening hypothesis (see Fig. 3B). Hence, this study showed that participants can strengthen the cued item without necessarily changing the accessibility of the noncued items.

Finally, there is evidence that retro-cues counteract the detrimental effect of reducing attention to the memory array during encoding (Vandenbroucke, Sligte, \& Lamme, 2011). Vandenbroucke et al. showed that WM performance suffers when, at encoding, attention is diverted from the memory array by, for example, creating uncertainty about which display is memory-relevant, or by asking people to perform another task concurrently with encoding of the memory array. In these conditions, presenting a retro-cue during the retention

\footnotetext{
$\overline{{ }^{8} \text { These results }}$ are only tentatively informative, because those authors did not provide any means of assessing the amount of support for the null hypothesis.
} 
interval of the memory task reduces the deleterious effect of distraction during encoding. This finding suggests that reduced attention during encoding results in the formation of relatively weak memory traces, which can be strengthened by focusing attention on the relevant item prior to test.

To conclude, the strengthening of WM representations by focused attention is a likely cause of the retro-cue benefit.

\section{H5. Retrieval head start}

We started this section by pointing out a conundrum: In many retro-cue experiments, a probe stimulus is presented in the nocue condition at the same time as the retro-cue is presented in the retro-cue condition, and the probe has more information to offer than the retro-cue, yet it yields worse performance. Perhaps, then, the retro-cue benefit is less about the information the retro-cue provides, and more about what it temporarily withholds. The last two hypotheses about the causes of the retro-cue benefit that we will consider state that access to visual WM is improved by delaying part of the information provided at test. The retro-cue delays the (potentially interfering) visual input of the test (e.g., the memory probe; or the continuous response scale). Moreover, it also delays all of the cognitive operations related to decision making (response selection and response execution), but leaves the opportunity to retrieve the cued item intact.

First, let's consider the contribution of delaying decision making. The retro-cue indicates the item that participants have to retrieve from WM, but it delays any other form of cognitive processing. Hence, participants have more undisrupted time to retrieve the target information from WM. How can this be helpful? Souza et al. (2016) argued that retrieval can be conceived as the gradual accumulation of information about the target's features in the focus of attention, a process akin to the gradual accumulation of evidence in sequential-sampling models of (perceptual) decision making (e.g., Purcell et al., 2010; Ratcliff \& McKoon, 2008; Ratcliff \& Rouder, 1998; Teodorescu \& Usher, 2013; Usher \& McClelland, 2001). In these models, evidence accumulates gradually over time. A decision is made once the accumulated evidence reaches a threshold, with the threshold reflecting the compromise between speed and accuracy set by the individual. Applying the same logic to retrieval, prolonging the retrieval time can be expected to improve performance (up to a certain limit) because more evidence is sampled from memory (as is illustrated in Fig. 4A). We will call this explanation the retrieval head start hypothesis.

The retrieval head start hypothesis rests on the assumption that decision making in no-cue trials commences before retrieval has extracted all information from WM, so that decision making is based on incomplete accumulated information. Consequently, the decision might be unduly influenced by other sources of information than the target, including nontarget representations in WM as well as the visual stimuli present at test (e.g., probe or distracting stimuli). In contrast, a retro-cue gives retrieval a head start, ensuring more advanced accumulation of evidence from memory before decision making can begin.

One may wonder why enforcing a longer delay helps performance in nonspeeded tests. There are at least two possibilities. First, in nonspeeded tasks, participants need to set a threshold of evidence accumulation before responding, thereby controlling the speed-accuracy trade-off. This threshold can vary across trials and individuals, and nothing guarantees that it will optimize accuracy. Hence, enforcing a delay may help to increase the probability of optimal evidence accumulation. Second, it might not be optimal to immediately use all retrieved information as evidence toward one or another response, because in the initial stages of retrieval, information from irrelevant sources of information (e.g., nontarget items in WM) is likely to be retrieved alongside relevant information about the target feature. Temporally separating retrieval and decision making, as a retro-cue does, could give the retrieval process time to narrow down its output to the relevant information before feeding it into the decision process, thereby reducing the influence of misleading information on response selection.

To provide a first test of the retrieval head start hypothesis, Souza et al. (2016) created an intermediate condition between the no-cue and retro-cue conditions in a local-recognition task. In this intermediate condition, a probe was shown at the same point in time as in the no-cue condition, but decision making had to be delayed by the same amount of time that was provided to make use of the retro-cue (henceforth, the delayedselection condition; see Fig. 5). Comparing the delayedselection condition to the no-cue condition allowed for testing the contribution of delaying decision making to the retro-cue benefit. Delaying response selection yielded a benefit (albeit smaller than the retro-cue benefit) to visual WM. In a similar vein, Souza et al. (2016) further tested the retrieval head start hypothesis in a delayed color estimation task by removing the color wheel from the screen during a delay-hence, preventing participants from responding. Again, a benefit (albeit small) was observed in the delay condition. In sum, these experiments show that delaying decision making accounts for part of the retro-cue benefit. This evidence is consistent with the predictions of a retrieval head start hypothesis.

The idea that retro-cues give retrieval stages a head start is in line with the event-related potential (ERP) topographies elicited by the probe stimulus in the neutral versus retro-cue trials reported by Nobre et al. (2008). They observed a loadsensitive ERP component (which they termed the $N 3_{R S}$ ) related to the search through items in WM in neutral-cue trials. By contrast, this component was not elicited by the probe in retrocue trials. This suggests that by the time the probe was displayed in retro-cue trials, participants might already have 


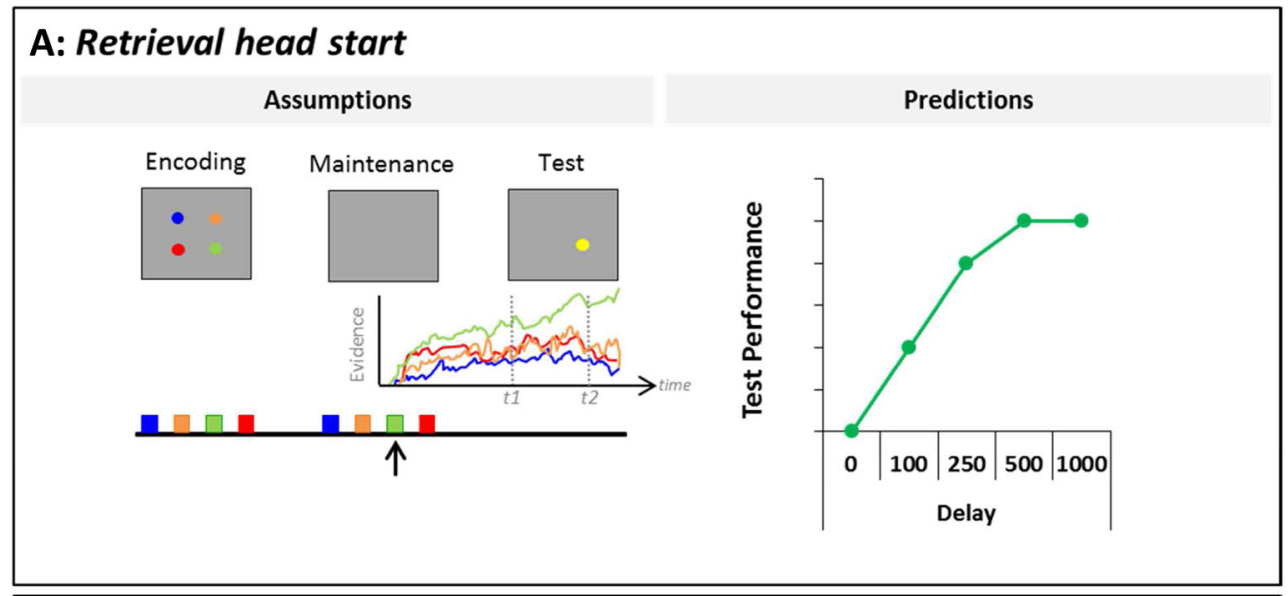

\section{B: Protection from perceptual interference}

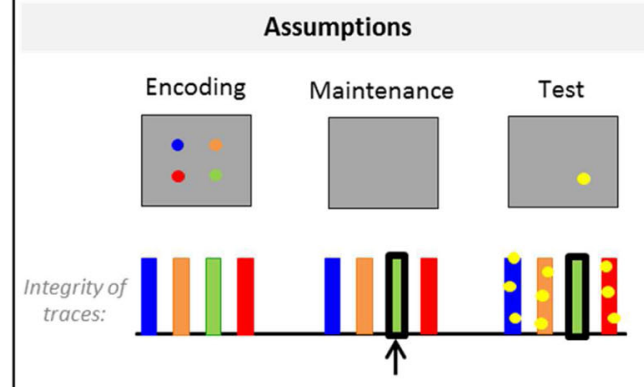

Predictions

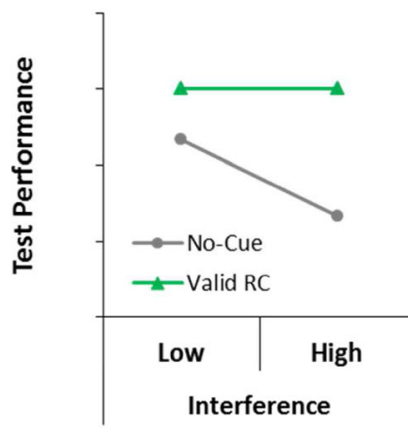

Fig. 4 Assumptions and predictions of retro-cue hypotheses. (A) Retrieval head start: Evidence for each item gradually accumulates in memory. The probability of retrieving an item is indexed by the relative evidence for this item (i.e., the height of its curve) in comparison to other alternatives (e.g., the heights of the remaining curves). Hence, the higher separation of the target (top line) in relation to the other items indicates the differential probability of retrieving the target. The retro-cue allows retrieval to start ahead of testing, yielding more time for evidence to accumulate in WM before decision than in the no-cue condition. This is simulated here by looking at the relative heights of the top line at time

finished retrieving the target item, providing tentative evidence in support of the retrieval head start hypothesis. This evidence is only tentative, though, because in this study they did not investigate whether the $\mathrm{N} 3_{\mathrm{RS}}$ was elicited by the retrocue (instead of the probe) and whether it was somehow modulated in retro-cue trials, showing the facilitation of retrieval that would be expected from the retrieval head start hypothesis.

\section{H6. Protection from perceptual interference}

The retro-cue not only delays response selection, it also delays the onset of interference from the visual input at test relative to the onset of the retrieval cue. Accordingly, one prominent hypothesis of the retro-cue benefit is that focused attention shields WM representations from perceptual interference points $\mathrm{t} 1$ (no cue) and $\mathrm{t} 2$ (retro-cue). (B) Protection from perceptual interference: Visual stimuli at test, such as a recognition probe (e.g., a yellow dot), interfere with memory representations, illustrated by the contamination of the bars representing noncued items by the probe's color (yellow dots in the online, color figure). This hypothesis entails the prediction that visual interference effects are reduced following a retro-cue because the retro-cued item is insulated from this contamination (illustrated here by a thick border, making it less permeable to the contamination). $\mathrm{RC}=$ retro-cue

(Makovski \& Jiang, 2007; Matsukura et al., 2007; Sligte et al., 2008). As is shown in Fig. 4B, such a hypothesis predicts that retro-cueing an item would make it more robust to interference from visual stimuli. The underlying assumption is that the WM representations in no-cue trials, as well as the noncued representations in retro-cue trials, are easily distorted by subsequent visual input. This leads to the prediction that after using the retro-cue to focus attention on one item, the cued item is protected from visual interference, whereas noncued representations remain unprotected, hence being impaired by interference.

Two lines of research speak to this hypothesis. First, some studies have assessed the impact of presenting irrelevant interfering information during the retention interval. Second, other studies have assessed how much interference is introduced by the visual stimuli in the 


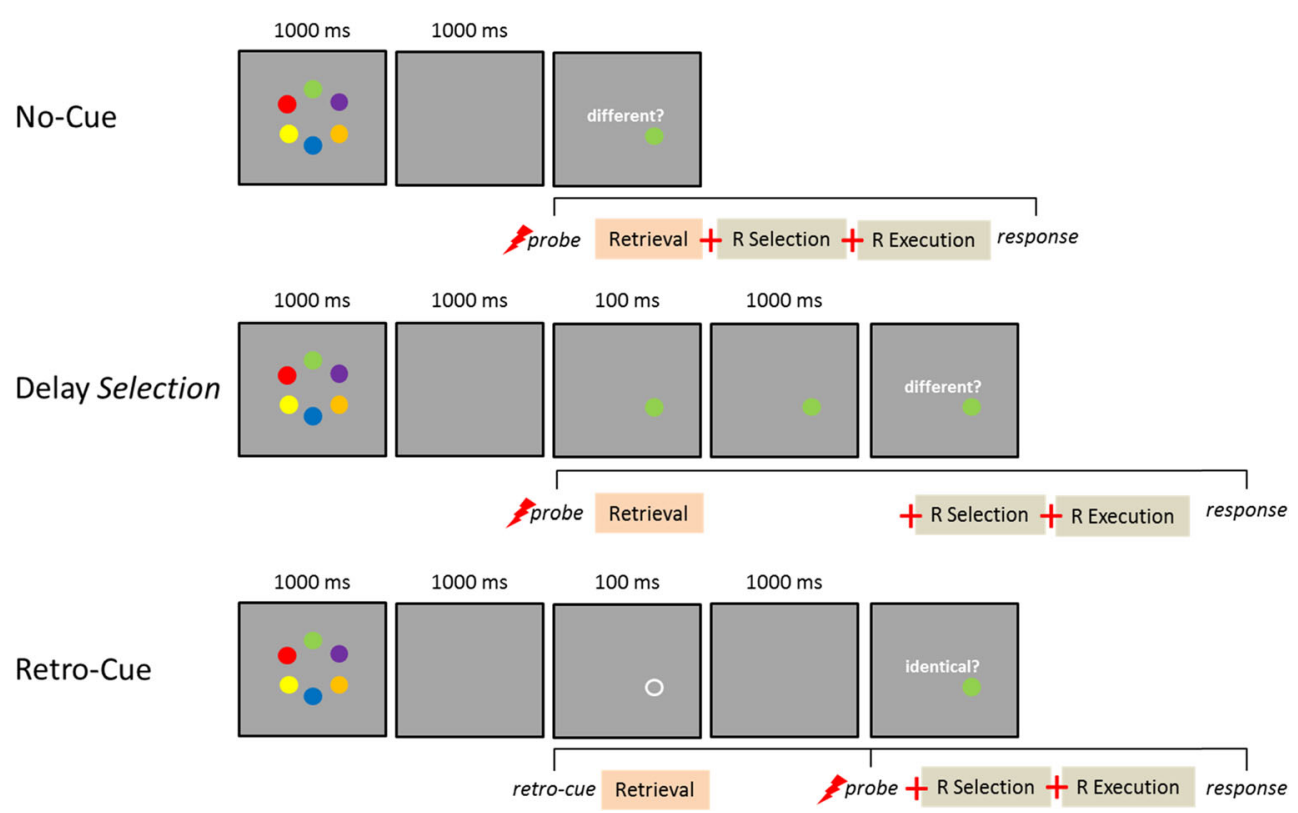

Fig. 5 Outline of no-cue, delayed-selection, and retro-cue conditions realized in Souza et al.'s (2016) study. In the no-cue condition, a probe and a question were presented simultaneously. Participants had to retrieve the item at the probe location, compare it to the probe, and answer the question displayed onscreen by pressing a "yes" or a "no" button. In the delayed-selection condition, the probe was shown at the same point in

test display, and how retro-cues can protect WM representations from it.

The first of these approaches tests the prediction that irrelevant visual input impairs memory for the target item less after a retro-cue to that item than in the absence of a retro-cue (Fig. 4B). Two studies have shown that presenting an irrelevant visual mask during the retention interval (after the retro-cue in retro-cue trials) yields results matching these predictions (Makovski \& Jiang, 2007; van Moorselaar, Gunseli, et al., 2015). In three other studies, interference was manipulated by presenting a secondary task during the retention interval — essentially creating a dual-task condition (Hollingworth \& Maxcey-Richard, 2013; Makovski \& Pertzov, 2015; Rerko et al., 2014). Makovski and Pertzov introduced an even-odd digit classification task during the retention interval of a delayed-estimation task, and observed that retro-cue benefits were significantly larger under the dual-task condition, as is predicted by the protection-frominterference hypothesis. Hollingworth and Maxcey-Richard as well as Rerko et al. also observed a numerical trend in this direction in local recognition; however, the Cueing $\times$ DualTask interaction was not significant, hence not providing evidence that could be used to either support or reject this hypothesis. All in all, studies testing for protection from irrelevant visual interference suggest that retro-cues can protect WM representation from interference by irrelevant visual input. time as in the no-cue condition (and, hence, could already start interfering with memory, as indicated by the lightning symbol), but response selection and execution had to be postponed until the question was displayed. In the retro-cue condition, the cue allowed retrieval to start, but interference from both the probe and decision making were postponed

The second approach to testing the protection-frominterference hypothesis is concerned with perceptual interference arising from the process of testing memory. Attending to the test display is required in order to complete the memory task, but at the same time, this introduces visual input that can harm memory. Retro-cues can serve to protect memory from this deleterious effect. Evidence in this direction has been provided by Makovski, Watson, Koutstaal, and Jiang (2010). These authors compared performance in a localrecognition task (single-probe display) with performance in a two-alternative forced choice task (two-probe display) with and without a retro-cue. Performance was overall lower with the two-probe display test, but retrocues reduced this disadvantage.

Souza et al. (2016) investigated the role of protection from visual interference in local-recognition and delayedestimation tasks. In recognition, visual interference arises from the memory probe. The retro-cue delays onset of the probe, giving focused attention time to make the cued item robust enough to withstand distortion. The retro-cue also postpones other processes involved in decision making, as we discussed above. To test separately the contributions of delaying decision and of delaying probe interference, Souza et al. (2016) created a delayed-selection condition that was inbetween the classical no-cue and retro-cue conditions (see Fig. 5). In this condition, a probe was shown at the same point in time as the retro-cue, but participants were forced to delay decision making for the same amount of time as in the retro- 
cue condition. Performance in the retro-cue condition was better than in this delay condition, indicating unambiguously that delaying probe interference contributes to the retro-cue benefit in this task, over and above the benefit from delaying the decision process.

Interference from the test display can also impair memory in the delayed-estimation paradigm. In a color delayedestimation task, a color wheel is shown at test. Souza et al. (2016) showed that the color wheel interferes with memory: When attending to the location of the target of recall, participants attend as well to the portion of the color wheel that is spatially close to it. Attending to this location attracts responses to spatially close colors in the color wheel, leading to a memory distortion effect that Souza et al. (2016) termed wheel attraction. The wheel attraction effect disappeared when the onset of the color wheel was delayed relative to the onset of the cue, and when the color wheel was replaced by a gray wheel. These results show that color information presented at test interferes with memory for colors, and that attention can be used to protect memory from this interference.

Taken together, there is strong evidence that part of the retro-cue benefit is due to protection of the focused WM representation from perceptual interference. For the purpose of this review, we treated the protection-from-interference hypothesis as a separate hypothesis, but we note that protection from perceptual interference could be a by-product of strengthening a focused representation in WM, or of a head start for retrieving that representation before the onset of interfering information. The two possibilities could be distinguished by a sequential-cueing paradigm in which first item $\mathrm{A}$, then item $\mathrm{B}$ is retro-cued, with the instruction that the lastcued item will be the most likely target. Strengthening of an item A in WM persists after attention focuses on another item B. Therefore, if strengthening underlies protection from perceptual interference, a visual mask following the second retrocue should affect item A (and item B) less than a noncued item C. In contrast, after the second retro-cue, only item $\mathrm{B}$ is given a head start for retrieval, whereas there is no reason to retrieve item $\mathrm{A}$ at all at this point in time. Therefore, if protection from perceptual interference is a by-product of having retrieved the item before the onset of interference, the effect of a visual mask should be reduced only for item B, not for A.

\section{Fragile/robust WM stores}

A discussion of explanations of the retro-cue effect would be incomplete without considering the hypothesis of multiple visual memory stores, advanced by Sligte, Lamme, and colleagues (Sligte et al., 2008; Sligte et al., 2010): Items selectively attended at encoding yield robust representations (robust WM store). These representations are protected from decay and from interference, and underlie performance in traditional tests of visual WM (e.g., in postcue or no-cue trials).
Items coarsely attended at encoding yield fragile traces (fragile WM store). These fragile representations are highly susceptible to interference from visual input presented at the same location and in the same feature dimension as the memoranda (Pinto et al., 2013; Sligte et al., 2008). Hence, a probe presented in the same location as the target - as in local-recognition or change discrimination tasks - erases any fragile representation of the target, so that performance can only rely on the contents of the robust WM store. When a fragile representation is attended during the retention interval-for instance, in response to a retro-cue - it is turned into a robust representation in WM, and thereby can contribute to performance. Finally, for nonattended items, only an iconic trace persists for $\sim 500 \mathrm{~ms}$ (Sligte et al., 2008; Sligte et al., 2010; Sperling, 1960). This iconic store has a high capacity, and quickly orienting attention to their fleeting representations allows their transfer to WM, hence dramatically increasing capacity estimates (Schmidt, Vogel, Woodman, \& Luck, 2002; Sligte et al., 2008; Sligte et al., 2010).

The theory of multiple visual stores makes assumptions and predictions that go substantially beyond an explanation of the retro-cue effect (Sligte, Scholte, \& Lamme, 2009; Vandenbroucke et al., 2011, 2015). A full evaluation of its merits also goes beyond the scope of this review. Here we focus on how the multiple-store theory explains the retro-cue effect. The explanation is to a large part a version of H6: Retrocued items are encoded in robust WM, and are thereby protected against interference from subsequent visual input. Unlike our general formulation of $\mathrm{H} 6$, which assumes that perceptual interference can arise from a broad range of sources, the multiple-store theory is committed to the assumption that only perceptual input in the same location as the memory items disrupts the fragile representations of these items (Pinto et al., 2013; Sligte et al., 2008). Therefore, the multiple-store theory in its current form cannot easily explain the retro-cue benefit in the delayed-estimation paradigm, in which no probe is presented at the location of the target. This problem could be overcome by assuming that fragile visual short-term memory can also be disrupted by visual input in other locations than the target, such as the onset of a color wheel in the delayed-estimation task (Souza et al., 2016).

One unique prediction of the multiple-store theory is that retro-cues will only be beneficial when presented prior to the onset of visual interference, which is assumed to entirely erase fragile representations so that a subsequent retro-cue cannot resuscitate them. The evidence for this strong prediction is currently mixed. Sligte et al. (2008) and Pinto et al. (2013) observed that presenting a visual mask consisting of similar items in the same location as the memoranda prior to onset of the retro-cue abolished the retro-cue benefit. In contrast, Makovski (2012) observed retro-cue benefits even when a visual mask (multicolored dots presented at the same location as the color memoranda) was displayed prior to the retro-cue. 
In sum, the multiple-store theory provides a mechanistic basis for the hypothesis that retro-cues protect memory representations from perceptual interference, and as such, the empirical support for this hypothesis (H6) also supports the multiple-store theory. At the same time, our discussion of H6 has shown that it does not offer a complete explanation of the retro-cue effect on its own, and the same is true for the multiple-store theory. Moreover, H6 does not require the assumption of multiple stores: A theory of visual WM could assume a single WM store in which representations can vary on a continuum of robustness, and attending to a representation increases its robustness, thereby protecting it from perceptual interference.

\section{Interim summary}

We have reviewed evidence speaking to six hypotheses about causes of the retro-cue effect; here we summarize our conclusions about their viability.

1. Protection from decay might contribute to the retro-cue effect in experiments comparing the retro-cue condition to a no-cue long baseline, but the hypothesis is contingent on the assumption that decay contributes to forgetting in visual WM, for which evidence is mixed at best.

2. Prioritization for comparison could contribute to the retrocue benefit only in tasks that require search through multiple memory items. Most retro-cue tasks constrain the comparison to a single item in both retro-cue and no-cue trials, making this explanation nonappealing.

3. Highly reliable retro-cues indicate the relevant item for the test, and render noncued items irrelevant. Removing irrelevant information frees WM capacity. The evidence supporting removal of noncued items from WM converges with the finding that visual WM's contents can be flexibly discarded to encode new, more up-to-date information on an item basis (Kessler et al., 2015), and with evidence for the removal of outdated information as a component of updating of verbal WM (Ecker, Lewandowsky, \& Oberauer, 2014; Ecker, Oberauer, \& Lewandowsky, 2014).

4. According to the strengthening/refreshing hypothesis, attention can be used to cumulatively strengthen representations in WM, thereby augmenting, in an incremental fashion, their accessibility to levels higher than the one obtained just after encoding. There is compelling evidence that strengthening contributes to the retro-cue benefit. The relative contributions of refreshing and removal as mechanisms of the retro-cue benefit seems to depend critically on the reliability of the retro-cue. The latter finding underscores the voluntary, controlled use of the information provided by the retro-cue to modulate WM maintenance.
5. The retro-cue benefit is in part due to a head start for retrieval. Evidence for this hypothesis comes from the observation that merely delaying decision making improves performance on visual WM tests, even in the presence of perceptual interference. This finding shows that WM performance is constrained not only by limitations during maintenance but also at decision stages, due to nonoptimal selection of the information in WM.

6. Retro-cues make the representation of the cued item more robust to subsequent visual interference. The studies investigating protection from interference have shown that capacity limits in visual WM arise in part from interference from subsequent visual input (either irrelevant or relevant to the memory test). Retro-cues help stabilize representations to survive interference from that input.

In a nutshell, these results show how one can get the most out of visual WM, indicating possible avenues to explore when trying to bypass the severe capacity limitation that has been a hallmark of visual WM.

\section{Outlook and conclusion}

Research on retro-cue effects has provided important insights into the mechanisms of visual WM and of attention to its contents: The representations in visual WM are not as robust

Table 2 Questions for future research

1 What is selected by the retro-cue (e.g., item, context, or item-context bindings)?

2 What is the mechanism supporting the state of heightened accessibility and robustness achieved for the retro-cued item?

3 What is the relation between WM capacity measures in standard conditions (no-cue baseline) and performance in retro-cue trials? So far we do not know whether people with high capacity benefit more or less from the retro-cue than do low-capacity individuals. This information might be helpful in relating the retro-cue effect to models of capacity limitations in WM.

4 How does the retro-cue effect relate to the control of attention in other domains? We are not aware of studies that have tried to relate the retro-cue effect to other measures of the efficiency of attention control in the perceptual domain (e.g., cueing advantages in perceptual tasks), or even to other effects ascribed to the focus of attention in WM, such as object-switch costs (Garavan, 1998; Oberauer, 2003) or the last-item recency effect (McElree, 2001; Nee \& Jonides, 2011).

5 Does focusing attention on the retro-cued item change its status in long-term memory (LTM)? Relatedly, do LTM representations mediate the retro-cue effect? A first hint that retro-cueing an item does not change its status in LTM was provided by the recent study by LaRocque et al. (2015). However, more studies will be needed to settle this issue.

6 Do the different processes contributing to the retro-cue benefit (e.g., strengthening vs. removal) follow different developmental trajectories? 
as has previously been thought, and much of visual WM's capacity limitations may arise from interference (interitem interference and perceptual interference) and incomplete retrieval. Simply put, the retro-cue effect has shown that traditional tests substantially underestimate the information available in memory. In the WM literature, it has usually been assumed that performance on WM tests is more or less a direct function of the limitations of encoding and storage. Hence, the precision and number of items stored in memory are assumed to have the values estimated in traditional memory test conditions. The retro-cue effect shows that this is not the case. Representational quality could be higher, the number of items accessible from memory could be higher, or both, because many of the limitations we observe in traditional tests arise at retrieval and decision stages. For example, an increase in the estimated representational quality (precision) following a retro-cue does not mean that the retro-cue yielded an information gain from nowhere, but that some precision is lost through testing memory. The retro-cue prevents this loss from happening, surprisingly showing higher memory precision when attention is focused on the target item prior to testing.

Research on the retro-cue effect has shown that attention can be allocated in a controlled fashion to the individual contents of visual WM, flexibly modulating them along at least four routes: Representations of individual items are strengthened, stabilized against perceptual interference, and selected for retrieval, and nonattended representations can be removed when they are unlikely to be needed soon. The retro-cue paradigm has proven exceptionally fruitful for investigating the interplay of WM and attention, and it is likely to continue to inspire empirical examinations of the boundary conditions of retro-cue effects, hypotheses about their underlying mechanisms, and predictions following from them. In Table 2, we propose a list of questions that might guide future research using the retro-cue paradigm.

As is the case in many maturing fields of research in cognitive psychology, the richness of the hypotheses about the nature of retro-cue effects and of the empirical findings speaking to them is beginning to weigh down on our ability to navigate the space of theoretical and empirical possibilities. The complexity of theoretical assumptions taxes the WM capacity of researchers trying to derive predictions from them. In this review, we handled this complexity by selectively focusing attention on hypotheses individually, but we acknowledge that this can only be an intermediate step. Most of the hypotheses we discussed are not mutually exclusive, and the evidence we reviewed strongly suggests that at least four of them contribute to retro-cue effects. Future theorizing about the role of attention in visual WM therefore will have to consider how these processes, and potentially others, work together.

At this juncture, computational modeling can help as a cognitive prosthesis (Farrell \& Lewandowsky, 2010) for building and evaluating more complex theories about the mechanisms and processes underlying attention to the contents of visual WM. Several computational models already exist for visual WM (Bays, 2014; Matthey, Bays, \& Dayan, 2015; Swan \& Wyble, 2014; van den Berg, Shin, Chou, George, \& Ma, 2012). We recently proposed a computational model of attention to items in verbal WM (Oberauer, 2013; Oberauer, Souza, Druey, \& Gade, 2013) that explains the effects of switching attention between items in WM and of swapping entire memory sets into and out of WM. Integrating the principles and mechanisms of these two lines of modeling might be a fruitful way toward a computational model of attention to the contents of (visual) WM.

Author note This project was supported by a grant from the Forschungskredit (Project No. FK-13-083) to A.S.S. and by a grant from the Swiss National Science Foundation (Project No. 126766/1) to K.O.

\section{References}

Astle, D. E., Nobre, A. C., \& Scerif, G. (2012). Attentional control constrains visual short-term memory: Insights from developmental and individual differences. Quarterly Journal of Experimental Psychology, 65, 277-294. doi:10.1080/17470218.2010.492622

Astle, D. E., Summerfield, J., Griffin, I., \& Nobre, A. C. (2012). Orienting attention to locations in mental representations. Attention, Perception, \& Psychophysics, 74, 146-162. doi: 10.3758/s13414-011-0218-3

Backer, K. C., \& Alain, C. (2012). Orienting attention to sound object representations attenuates change deafness. Journal of Experimental Psychology: Human Perception and Performance, 38, 1554-1566.

Backer, K. C., Binns, M. A., \& Alain, C. (2015). Neural dynamics underlying attentional orienting to auditory representations in shortterm memory. Journal of Neuroscience, 35, 1307-1318.

Bays, P. M. (2014). Noise in neural populations accounts for errors in working memory. Journal of Neuroscience, 34, 3632-3645.

Bays, P. M., Catalao, R. F. G., \& Husain, M. (2009). The precision of visual working memory is set by allocation of a shared resource. Journal of Vision, 9(10), 7:1-11. doi:10.1167/9.10.7

Bays, P. M., \& Husain, M. (2008). Dynamic shifts of limited working memory resources in human vision. Science, 321, 851-854. doi:10.1126/science. 1158023

Beck, M. R., \& van Lamsweerde, A. E. (2011). Accessing long-term memory representations during visual change detection. Memory \& Cognition, 39, 433-446. doi:10.3758/s13421-010-0033-4

Berryhill, M. E., Richmond, L. L., Shay, C. S., \& Olson, I. R. (2012). Shifting attention among working memory representations: Testing cue type, awareness, and strategic control. Quarterly Journal of Experimental Psychology, 65, 426-438.

Brown, G. D. A., Neath, I., \& Chater, N. (2007). A temporal ratio model of memory. Psychological Review, 114, 539-576. doi:10.1037/0033-295X.114.3.539

Brown, G. D. A., Sala, S. D., Foster, J. K., \& Vousden, J. I. (2007). Amnesia, rehearsal, and temporal distinctiveness models of recall. Psychonomic Bulletin \& Review, 14, 256-260.

Bull, R., Espy, K. A., \& Wiebe, S. A. (2008). Short-term memory, working memory, and executive functioning in preschoolers: Longitudinal predictors of mathematical achievement at age 7 years. Developmental Neuropsychology, 33, 205-228.

Carrasco, M. (2011). Visual attention: The past 25 years. Vision Research, 51, 1484-1525. doi:10.1016/j.visres.2011.04.012 
Chun, M. M., Golomb, J. D., \& Turk-Browne, N. B. (2011). A taxonomy of external and internal attention. Annual Review of Psychology, 62, 73-101. doi:10.1146/annurev.psych.093008.100427

Chun, M. M., \& Johnson, M. K. (2011). Memory: Enduring traces of perceptual and reflective attention. Neuron, 72, 520-535.

Conway, A. R. A., Kane, M. J., \& Engle, R. W. (2003). Working memory capacity and its relation to general intelligence. Trends in Cognitive Sciences, 7, 547-552. doi:10.1016/j.tics.2003.10.005

Cowan, N. (2010). The magical mystery four: How is working memory capacity limited, and why? Current Directions in Psychological Science, 19, 51-57. doi:10.1177/0963721409359277

Daneman, M., \& Carpenter, P. A. (1980). Individual differences in working memory and reading. Journal of Verbal Learning and Verbal Behavior, 19, 450-466. doi:10.1016/S0022-5371(80)90312-6

Delvenne, J.-F., Cleeremans, A., \& Laloyaux, C. (2010). Feature bindings are maintained in visual short-term memory without sustained focused attention. Experimental Psychology, 57, 108-116. doi:10.1027/1618-3169/a000014

Delvenne, J.-F., \& Holt, J. L. (2012). Splitting attention across the two visual fields in visual short-term memory. Cognition, 122, 258-263.

Duarte, A., Hearons, P., Jiang, Y., Delvin, M. C., Newsome, R. N., \& Verhaeghen, P. (2013). Retrospective attention enhances visual working memory in the young but not the old: An ERP study. Psychophysiology, 50, 465-476.

Ecker, U. K. H., Lewandowsky, S., \& Oberauer, K. (2014). Removal of information from working memory: A specific updating process. Journal of Memory and Language, 74, 77-90. doi:10.1016/j.jml. 2013.09.003

Ecker, U. K. H., Oberauer, K., \& Lewandowsky, S. (2014). Working memory updating involves item-specific removal. Journal of Memory and Language, 74, 1-15. doi:10.1016/j.jml.2014.03.006

Egeth, H. E., \& Yantis, S. (1997). Visual attention: Control, representation, and time course. Annual Review of Psychology, 48, 269-297. doi:10.1146/annurev.psych.48.1.269

Farrell, S., \& Lewandowsky, S. (2010). Computational models as aids to better reasoning in psychology. Current Directions in Psychological Science, 19, 329-335.

Garavan, H. (1998). Serial attention within working memory. Memory \& Cognition, 26, 263-276. doi:10.3758/BF03201138

Gazzaley, A., \& Nobre, A. C. (2012). Top-down modulation: Bridging selective attention and working memory. Trends in Cognitive Sciences, 16, 129-135. doi:10.1016/j.tics.2011.11.014

Gilchrist, A. L., Duarte, A., \& Verhaeghen, P. (2016). Retrospective cues based on object features improve visual working memory performance in older adults. Neuropsychology, Development, and Cognition, Section B, 23, 184-195. doi:10.1080/13825585.2015.1069253

Gorgoraptis, N., Catalao, R. F. G., Bays, P. M., \& Husain, M. (2011). Dynamic updating of working memory resources for visual objects. Journal of Neuroscience, 31, 8502-8511. doi: 10.1523/JNEUROSCI.0208-11.2011

Gözenman, F., Tanoue, R. T., Metoyer, T., \& Berryhill, M. E. (2014). Invalid retro-cues can eliminate the retro-cue benefit: Evidence for a hybridized account. Journal of Experimental Psychology. Human Perception and Performance, 40, 1748-1754.

Gressmann, M., \& Janczyk, M. (2016). The (un)clear effects of invalid retro-cues. Frontiers in Psychology, 7, 244. doi:10.3389/fpsyg. 2016.00244

Griffin, I. C., \& Nobre, A. C. (2003). Orienting attention to locations in internal representations. Journal of Cognitive Neuroscience, 15, 1176-1194. doi:10.1162/089892903322598139

Gunseli, E., van Moorselaar, D., Meeter, M., \& Olivers, C. N. L. (2015). The reliability of retro-cues determines the fate of noncued visual working memory representations. Psychonomic Bulletin \& Review, 22, 1334-1341. doi:10.3758/s13423-014-0796-x
Heuer, A., \& Schubö, A. (2016). Feature-based and spatial attentional selection in visual working memory. Memory \& Cognition. Advance online publication. doi:10.3758/s13421-015-0584-5

Hollingworth, A. (2003). Failures of retrieval and comparison constrain change detection in natural scenes. Journal of Experimental Psychology: Human Perception and Performance, 29, 388-403. doi:10.1037/0096-1523.29.2.388

Hollingworth, A., \& Maxcey-Richard, A. M. (2013). Selective maintenance in visual working memory does not require sustained visual attention. Journal of Experimental Psychology: Human Perception and Performance, 39, 1047-1058. doi:10.1037/a0030238

Irwin, D. E., \& Thomas, L. E. (2008). Visual sensory memory. In S. J. Luck \& A. Hollingworth (Eds.), Visual memory (pp. 9-41). New York, NY: Oxford University Press.

Janczyk, M., \& Berryhill, M. E. (2014). Orienting attention in visual working memory requires central capacity: Decreased retro-cue effects under dual-task conditions. Attention, Perception, \& Psychophysics, 76, 715-724. doi:10.3758/s13414-013-0615-x

Johnson, M. K. (1992). MEM: Mechanisms of recollection. Journal of Cognitive Neuroscience, 4, 268-280. doi:10. 1162/jocn.1992.4.3.268

Katus, T., Andersen, S. K., \& Müller, M. M. (2012a). Maintenance of tactile short-term memory for locations is mediated by spatial attention. Biological Psychology, 89, 39-46. doi:10.1016/j.biopsycho. 2011.09.001

Katus, T., Andersen, S. K., \& Müller, M. M. (2012b). Nonspatial cueing of tactile STM causes shift of spatial attention. Journal of Cognitive Neuroscience, 24, 1596-1609. doi:10.1162/jocn a 00234

Katus, T., Andersen, S. K., \& Müller, M. M. (2014). Common mechanisms of spatial attention in memory and perception: A tactile dual-task study. Cerebral Cortex, 24, 707-718. doi: 10.1093/cercor/bhs350

Kessler, Y., Rac-Lubashevsky, R., Lichtstein, C., Markus, H., Simchon, A., \& Moscovitch, M. (2015). Updating visual working memory in the change detection paradigm. Journal of Vision, 15(9), 18. doi: $10.1167 / 15.9 .18$

Kuo, B.-C., Stokes, M. G., \& Nobre, A. C. (2012). Attention modulates maintenance of representations in visual short-term memory. Journal of Cognitive Neuroscience, 24, 51-60. doi: 10.1162/jocn_a_00087

Kuo, B.-C., Yeh, Y.-Y., Chen, A. J.-W., \& D’Esposito, M. (2011). Functional connectivity during top-down modulation of visual short-term memory representations. Neuropsychologia, 49, $1589-1596$.

Landman, R., Spekreijse, H., \& Lamme, V. A. F. (2003). Large capacity storage of integrated objects before change blindness. Vision Research, 43, 149-164. doi:10.1016/S0042-6989(02)00402-9

Landman, R., Spekreijse, H., \& Lamme, V. A. F. (2004). The role of figure-ground segregation in change blindness. Psychonomic Bulletin \& Review, 11, 254-261.

LaRocque, J. J., Eichenbaum, A. S., Starrett, M. J., Rose, N. S., Emrich, S. M., \& Postle, B. R. (2015). The short- and long-term fates of memory items retained outside the focus of attention. Memory \& Cognition, 43, 453-468. doi:10.3758/s13421-014-0486-y

LaRocque, J. J., Lewis-Peacock, J. A., Drysdale, A. T., Oberauer, K., \& Postle, B. R. (2013). Decoding attended information in short-term memory: An EEG study. Journal of Cognitive Neuroscience, 25, 127-142. doi:10.1162/jocn_a 00305

Lawrence, M. A. (2010). Estimating the probability and fidelity of memory. Behavior Research Methods, 42, 957-968. doi:10.3758/BRM. 42.4.957

Lepsien, J., Griffin, I. C., Devlin, J. T., \& Nobre, A. C. (2005). Directing spatial attention in mental representations: Interactions between attentional orienting and working-memory load. NeuroImage, 26, 733-743. doi:10.1016/j.neuroimage.2005.02.026 
Lepsien, J., \& Nobre, A. C. (2007). Attentional modulation of object representations in working memory. Cerebral Cortex, 17, 20722083. doi:10.1093/cercor/bhl116

Lepsien, J., Thornton, I., \& Nobre, A. C. (2011). Modulation of workingmemory maintenance by directed attention. Neuropsychologia, 49, 1569-1577. doi:10.1016/j.neuropsychologia.2011.03.011

Lewis-Peacock, J. A., Drysdale, A. T., Oberauer, K., \& Postle, B. R. (2012). Neural evidence for a distinction between short-term memory and the focus of attention. Journal of Cognitive Neuroscience, 24, 61-79.

Li, Q., \& Saiki, J. (2014). The effects of sequential attention shifts within visual working memory. Frontiers in Psychology, 5, 965. doi: 10.3389/fpsyg.2014.00965

Li, Q., \& Saiki, J. (2015). Different effects of color-based and locationbased selection on visual working memory. Attention, Perception, \& Psychophysics, 77, 450-463. doi:10.3758/s13414-014-0775-3

Luck, S. J., \& Vogel, E. K. (1997). The capacity of visual working memory for features and conjunctions. Nature, 390, 279-281. doi: $10.1038 / 36846$

Makovski, T. (2012). Are multiple visual short-term memory storages necessary to explain the retro-cue effect? Psychonomic Bulletin \& Review, 19, 470-476. doi:10.3758/s13423-012-0235-9

Makovski, T., \& Jiang, Y. V. (2007). Distributing versus focusing attention in visual short-term memory. Psychonomic Bulletin \& Review, 14, 1072-1078. doi:10.3758/BF03193093

Makovski, T., \& Pertzov, Y. (2015). Attention and memory protection: Interactions between retrospective attention cueing and interference. Quarterly Journal of Experimental Psychology, 68, 1735-1743. doi: 10.1080/17470218.2015.1049623

Makovski, T., Sussman, R., \& Jiang, Y. V. (2008). Orienting attention in visual working memory reduces interference from memory probes. Journal of Experimental Psychology: Learning, Memory, and Cognition, 34, 369-380. doi:10.1037/0278-7393.34.2.369

Makovski, T., Watson, L. M., Koutstaal, W., \& Jiang, Y. V. (2010). Method matters: Systematic effects of testing procedure on visual working memory sensitivity. Journal of Experimental Psychology: Learning, Memory, and Cognition, 36, 14661479. doi:10.1037/a0020851

Matsukura, M., Cosman, J. D., Zachary, J. J. R., Vatterott, D. B., \& Vecera, S. P. (2014). Location-specific effects of attention during visual short-term memory maintenance. Journal of Experimental Psychology: Human Perception and Performance, 40, 1103-1116.

Matsukura, M., Luck, S. J., \& Vecera, S. P. (2007). Attention effects during visual short-term memory maintenance: Protection or prioritization? Perception \& Psychophysics, 69, 1422-1434. doi:10.3758/BF03192957

Matsukura, M., \& Vecera, S. P. (2015). Selection of multiple cued items is possible during visual short-term memory maintenance. Attention, Perception, \& Psychophysics, 77, 1625-1646.

Matthey, L., Bays, P. M., \& Dayan, P. (2015). A probabilistic palimpsest model of visual short-term memory. PLoS Computational Biology, 11, e1004003. doi:10.1371/journal.pcbi.1004003

Maxcey, A. M., Fukuda, K., Song, W. S., \& Woodman, G. F. (2015). Using electrophysiology to demonstrate that cueing affects longterm memory storage over the short term. Psychonomic Bulletin \& Review, 22, 1349-1357.

Maxcey-Richard, A. M., \& Hollingworth, A. (2013). The strategic retention of task-relevant objects in visual working memory. Journal of Experimental Psychology: Learning, Memory, and Cognition, 39, 760-772. doi:10.1037/a0029496

McElree, B. (2001). Working memory and focal attention. Journal of Experimental Psychology: Learning, Memory, and Cognition, 27, 817-835. doi:10.1037/0278-7393.27.3.817

Mercer, T. (2014). The loss of short-term visual representations over time: Decay or temporal distinctiveness? Journal of Experimental Psychology: Human Perception and Performance, 40, 2281-2288.
Morey, C. C., \& Bieler, M. (2013). Visual short-term memory always requires general attention. Psychonomic Bulletin \& Review, 20, $163-170$.

Murray, A. M., Nobre, A. C., Clark, I. A., Cravo, A. M., \& Stokes, M. G. (2013). Attention restores discrete items to visual short-term memory. Psychological Science, 24, 550-556.

Myers, N. E., Walther, L., Wallis, G., Stokes, M. G., \& Nobre, A. C. (2015). Temporal dynamics of attention during encoding versus maintenance of working memory: Complementary views from event-related potentials and alpha-band oscillations. Journal of Cognitive Neuroscience, 27, 492-508. doi:10.1162/jocn_a_00727

Nee, D. E., \& Jonides, J. (2011). Dissociable contributions of prefrontal cortex and the hippocampus to short-term memory: Evidence for a 3-state model of memory. NeuroImage, 54, 1540-1548.

Nobre, A. C., Griffin, I. C., \& Rao, A. (2008). Spatial attention can bias search in visual short-term memory. Frontiers in Human Neuroscience, 1(4), 1-9. doi:10.3389/neuro.09.004.2007

Oberauer, K. (2001). Removing irrelevant information from working memory: A cognitive aging study with the modified Sternberg task. Journal of Experimental Psychology: Learning, Memory, and Cognition, 27, 948-957. doi:10.1037/0278-7393.27.4.948

Oberauer, K. (2002). Access to information in working memory: Exploring the focus of attention. Journal of Experimental Psychology: Learning, Memory, and Cognition, 28, 411-421. doi: 10.1037/0278-7393.28.3.411

Oberauer, K. (2003). Selective attention to elements in working memory. Experimental Psychology, 50, 257-269.

Oberauer, K. (2005). Control of the contents of working memory-A comparison of two paradigms and two age groups. Journal of Experimental Psychology: Learning, Memory, and Cognition, 31, 714-728. doi:10.1037/0278-7393.31.4.714

Oberauer, K. (2013). The focus of attention in working memory: From metaphors to mechanisms. Frontiers in Human Neuroscience, 7 , 673. doi:10.3389/fnhum.2013.00673

Oberauer, K., \& Hein, L. (2012). Attention to information in working memory. Current Directions in Psychological Science, 21, 164-169.

Oberauer, K., Souza, A. S., Druey, M. D., \& Gade, M. (2013). Analogous mechanisms of selection and updating in declarative and procedural working memory: Experiments and a computational model. Cognitive Psychology, 66, 157-211.

Olivers, C. N. L., Peters, J., Houtkamp, R., \& Roelfsema, P. R. (2011). Different states in visual working memory: When it guides attention and when it does not. Trends in Cognitive Sciences, 15, 327-334.

Pashler, H. (1994). Dual-task interference in simple tasks: Data and theory. Psychological Bulletin, 116, 220-244. doi:10. 1037/0033-2909.116.2.220

Pertzov, Y., Bays, P. M., Joseph, S., \& Husain, M. (2013). Rapid forgetting prevented by retrospective attention cues. Journal of Experimental Psychology: Human Perception and Performance, 39, 1224-1231. doi:10.1037/a0030947

Pinto, Y., Sligte, I. G., Shapiro, K. L., \& Lamme, V. A. F. (2013). Fragile visual short-term memory is an object-based and location-specific store. Psychonomic Bulletin \& Review, 20, 732-739. doi:10.3758/ s13423-013-0393-4

Posner, M. I. (1980). Orienting of attention. Quarterly Journal of Experimental Psychology, 32, 3-25. doi:10.1080/00335558008248231

Prinzmetal, W., Amiri, H., Allen, K., \& Edwards, T. (1998). Phenomenology of attention: I. Color, location, orientation, and spatial frequency. Journal of Experimental Psychology: Human Perception and Performance, 24, 261-282. doi:10.1037/00961523.24.1.261

Purcell, B. A., Heitz, R. P., Cohen, J. Y., Schall, J. D., Logan, G. D., \& Palmeri, T. J. (2010). Neurally constrained modeling of perceptual decision making. Psychological Review, 117, 1113-1143. doi: 10.1037/a0020311 
Ratcliff, R., \& McKoon, G. (2008). The diffusion decision model: Theory and data for two-choice decision tasks. Neural Computation, 20, 873-922. doi:10.1162/neco.2008.12-06-420

Ratcliff, R., \& Rouder, J. N. (1998). Modeling response times for twochoice decisions. Psychological Science, 9, 347-356. doi:10.1111/ 1467-9280.00067

Rensink, R. A. (2002). Change detection. Annual Review of Psychology, 53, 245-277. doi:10.1146/annurev.psych.53.100901.135125

Rerko, L., \& Oberauer, K. (2013). Focused, unfocused, and defocused information in working memory. Journal of Experimental Psychology: Learning, Memory, and Cognition, 39, 1075-1096. doi: $10.1037 / \mathrm{a} 0031172$

Rerko, L., Souza, A. S., \& Oberauer, K. (2014). Retro-cue benefits in working memory without sustained focal attention. Memory \& Cognition, 42, 712-728. doi:10.3758/s13421-013-0392-8

Ricker, T. J., \& Cowan, N. (2010). Loss of visual working memory within seconds: The combined use of refreshable and non-refreshable features. Journal of Experimental Psychology: Learning, Memory, and Cognition, 36, 1355-1368.

Ricker, T. J., \& Cowan, N. (2014). Differences between presentation methods in working memory procedures: A matter of working memory consolidation. Journal of Experimental Psychology: Learning, Memory, and Cognition, 40, 417-428.

Ricker, T. J., Spiegel, L. R., \& Cowan, N. (2014). Time-based loss in visual short-term memory is from trace decay, not temporal distinctiveness. Journal of Experimental Psychology: Learning, Memory, and Cognition, 40, 1510-1523.

Rouder, J. N., Morey, R. D., Morey, C. C., \& Cowan, N. (2011). How to measure working memory capacity in the change detection paradigm. Psychonomic Bulletin \& Review, 18, 324-330.

Schmidt, B. K., Vogel, E. K., Woodman, G. F., \& Luck, S. J. (2002). Voluntary and automatic attentional control of visual working memory. Perception \& Psychophysics, 64, 754-763. doi:10.3758/ BF03194742

Schneider, D., Mertes, C., \& Wascher, E. (2015). On the fate of non-cued mental representations in visuo-spatial working memory: Evidence by a retro-cuing paradigm. Behavioural Brain Research, 293, 114-124.

Serences, J. T., \& Yantis, S. (2006). Selective visual attention and perceptual coherence. Trends in Cognitive Sciences, 10, 38-45.

Shimi, A., Nobre, A. C., Astle, D. E., \& Scerif, G. (2014). Orienting attention within visual short-term memory: Development and mechanisms. Child Development, 85, 578-592.

Shipstead, Z., \& Engle, R. W. (2013). Interference within the focus of attention: Working memory tasks reflect more than temporary maintenance. Journal of Experimental Psychology: Learning, Memory, and Cognition, 39, 277-289. doi:10.1037/a0028467

Sligte, I. G., Scholte, H. S., \& Lamme, V. A. F. (2008). Are there multiple visual short-term memory stores? PLoS ONE, 3, e1699. doi: 10.1371/journal.pone.0001699

Sligte, I. G., Scholte, H. S., \& Lamme, V. A. F. (2009). V4 activity predicts the strength of visual short-term memory representations. Journal of Neuroscience, 29, 7432-7438. doi:10.1523/ JNEUROSCI.0784-09.2009

Sligte, I. G., Vandenbroucke, A. R. E., Scholte, H. S., \& Lamme, V. A. F. (2010). Detailed sensory memory, sloppy working memory. Frontiers in Psychology, 1, 175. doi:10.3389/fpsyg.2010.00175

Souza, A. S., \& Oberauer, K. (2015). Time-based forgetting in visual working memory reflects temporal distinctiveness, not decay. Psychonomic Bulletin \& Review, 22, 156-162.

Souza, A. S., Rerko, L., Lin, H.-Y., \& Oberauer, K. (2014). Focused attention improves working memory: Implications for flexibleresource and discrete-capacity models. Attention, Perception, \& Psychophysics, 76, 2080-2102.

Souza, A. S., Rerko, L., \& Oberauer, K. (2014). Unloading and reloading working memory: Attending to one item frees capacity. Journal of
Experimental Psychology: Human Perception and Performance, 40, 1237-1256.

Souza, A. S., Rerko, L., \& Oberauer, K. (2015). Refreshing memory traces: Thinking of an item improves retrieval from visual working memory. Annals of the New York Academy of Sciences, 1339, 20-31.

Souza, A. S., Rerko, L., \& Oberauer, K. (2016). Getting more from visual working memory: Retro-cues enhance retrieval and protect from visual interference. Journal of Experimental Psychology: Human Perception and Performance. Advance online publication. doi:10.1037/a0036331

Sperling, G. (1960). The information available in brief visual presentations. Psychological Monographs: General and Applied, 74(11, Whole No. 498), 1-29.

Stokes, M. G. (2015). "Activity-silent" working memory in prefrontal cortex: A dynamic coding framework. Trends in Cognitive Sciences, 19, 394-405.

Süß, H.-M., Oberauer, K., Wittmann, W. W., Wilhelm, O., \& Schulze, R. (2002). Working-memory capacity explains reasoning ability - and a little bit more. Intelligence, 30, 261-288.

Swan, G., \& Wyble, B. (2014). The binding pool: A model of shared neural resources for distinct items in visual working memory. Attention, Perception, \& Psychophysics, 76, 2136-2157.

Tamber-Rosenau, B. J., Esterman, M., Chiu, Y.-C., \& Yantis, S. (2011). Cortical mechanisms of cognitive control for shifting attention in vision and working memory. Journal of Cognitive Neuroscience, 23, 2905-2919.

Tanoue, R. T., \& Berryhill, M. E. (2012). The mental wormhole: Internal attention shifts without regard for distance. Attention, Perception, \& Psychophysics, 74, 1199-1215.

Teodorescu, A. R., \& Usher, M. (2013). Disentangling decision models: From independence to competition. Psychological Review, 120, 138. doi:10.1037/a0030776

Tombu, M., \& Jolicœur, P. (2003). A central capacity sharing model of dual-task performance. Journal of Experimental Psychology: Human Perception and Performance, 29, 3-18. doi:10.1037/00961523.29.1.3

Trapp, S., \& Lepsien, J. (2012). Attentional orienting to mnemonic representations: Reduction of load-sensitive maintenance-related activity in the intraparietal sulcus. Neuropsychologia, 50, 2805-2811.

Usher, M., \& McClelland, J. L. (2001). The time course of perceptual choice: The leaky, competing accumulator model. Psychological Review, 108, 550-592. doi:10.1037/0033-295X.111.3.757

van den Berg, R., Shin, H., Chou, W.-C., George, R., \& Ma, W. J. (2012). Variability in encoding precision accounts for visual short-term memory limitations. Proceedings of the National Academy of Sciences, 109, 8780-8785.

van Moorselaar, D., Gunseli, E., Theeuwes, J., \& Olivers, C. N. L. (2015). The time course of protecting a visual memory representation from perceptual interference. Frontiers in Human Neuroscience, 8, 1053. doi:10.3389/fnhum.2014.01053

van Moorselaar, D., Olivers, C. N. L., Theeuwes, J., Lamme, V. A. F., \& Sligte, I. G. (2015). Forgotten but not gone: Retro-cue costs and benefits in a double-cueing paradigm suggest multiple states in visual short-term memory. Journal of Experimental Psychology: Learning, Memory, and Cognition, 41, 1755-1763.

Vandenbroucke, A. R. E., Sligte, I. G., de Vries, J. G., Cohen, M. X., \& Lamme, V. A. F. (2015). Neural correlates of visual short-term memory dissociate between fragile and working memory representations. Journal of Cognitive Neuroscience, 27, 2477-2490.

Vandenbroucke, A. R. E., Sligte, I. G., Fahrenfort, J. J., Ambroziak, K. B., \& Lamme, V. A. F. (2012). Non-attended representations are perceptual rather than unconscious in nature. PLOS ONE, 7, e50042. doi:10.1371/journal.pone.0050042

Vandenbroucke, A. R. E., Sligte, I. G., \& Lamme, V. A. F. (2011). Manipulations of attention dissociate fragile visual short-term 
memory from visual working memory. Neuropsychologia, 49, $1559-1568$.

Wallis, G., Stokes, M., Cousijn, H., Woolrich, M., \& Nobre, A. C. (2015). Frontoparietal and cingulo-opercular networks play dissociable roles in control of working memory. Journal of Cognitive Neuroscience, 27, 2019-2034.

Wheeler, M. E., \& Treisman, A. M. (2002). Binding in short-term visual memory. Journal of Experimental Psychology: General, 131, 4864. doi:10.1037/0096-3445.131.1.48

Wilken, P., \& Ma, W. J. (2004). A detection theory account of change detection. Journal of Vision, 4(12), 11:1120-1135. doi:10.1167/4.12.11

Williams, M., Hong, S. W., Kang, M.-S., Carlisle, N. B., \& Woodman, G. F. (2013). The benefit of forgetting. Psychonomic Bulletin \& Review, 20, 348-355. doi:10.3758/s13423-012-0354-3

Williams, M., \& Woodman, G. F. (2012). Directed forgetting and directed remembering in visual working memory. Journal of Experimental Psychology: Learning, Memory, and Cognition, 38, 1206-1220.

Yantis, S. (2000). Goal-directed and stimulus-driven determinants of attentional control. In S. Monsell \& J. Driver (Eds.), Control of cognitive processes: Attention and performance XVIII (pp. 73 103). Cambridge, MA: MIT Press.

Yantis, S. (2008). The neural basis of selective attention cortical sources and targets of attentional modulation. Current Directions in Psychological Science, 17, 86-90. doi:10.1111/j.1467-8721.2008.00554.x

Zhang, W., \& Luck, S. J. (2008). Discrete fixed-resolution representations in visual working memory. Nature, 453, 233-235. doi:10.1038/ nature 06860

Zhang, W., \& Luck, S. J. (2009). Sudden death and gradual decay in visual working memory. Psychological Science, 20, 423-428. doi: 10.1111/j.1467-9280.2009.02322.x

Zokaei, N., Manohar, S., Husain, M., \& Feredoes, E. (2014). Causal evidence for a privileged working memory state in early visual cortex. Journal of Neuroscience, 34, 158-162. doi:10.1523/ JNEUROSCI.2899-13.2014

Zokaei, N., Ning, S., Manohar, S., Feredoes, E., \& Husain, M. (2014). Flexibility of representational states in working memory. Frontiers in Human Neuroscience, 8, 853. doi:10.3389/fnhum.2014.00853 